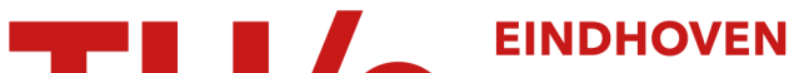 \\ UNIVERSITY OF \\ TECHNOLOGY
}

\section{Wave propagation in strain-softening plasticity}

\section{Citation for published version (APA):}

Sluys, L. J., \& Borst, de, R. (1993). Wave propagation in strain-softening plasticity. In D. Kolymbas (Ed.), Modern approaches to plasticity : proceedings of a workshop held in Horton, Greece, 12-16 June 1992 (pp. 411-447). Elsevier.

Document status and date:

Published: 01/01/1993

\section{Document Version:}

Publisher's PDF, also known as Version of Record (includes final page, issue and volume numbers)

\section{Please check the document version of this publication:}

- A submitted manuscript is the version of the article upon submission and before peer-review. There can be important differences between the submitted version and the official published version of record. People interested in the research are advised to contact the author for the final version of the publication, or visit the $\mathrm{DOI}$ to the publisher's website.

- The final author version and the galley proof are versions of the publication after peer review.

- The final published version features the final layout of the paper including the volume, issue and page numbers.

Link to publication

\section{General rights}

Copyright and moral rights for the publications made accessible in the public portal are retained by the authors and/or other copyright owners and it is a condition of accessing publications that users recognise and abide by the legal requirements associated with these rights.

- Users may download and print one copy of any publication from the public portal for the purpose of private study or research.

- You may not further distribute the material or use it for any profit-making activity or commercial gain

- You may freely distribute the URL identifying the publication in the public portal.

If the publication is distributed under the terms of Article 25fa of the Dutch Copyright Act, indicated by the "Taverne" license above, please follow below link for the End User Agreement:

www.tue.nl/taverne

Take down policy

If you believe that this document breaches copyright please contact us at:

openaccess@tue.nl

providing details and we will investigate your claim. 


\section{Wave Propagation in Strain-Softening Plasticity}

\section{L.J. Sluys and R. de Borst}

\section{Abstract}

Wave propagation in higher-order and rate-dependent strain-softening plasticity models is investigated analytically and numerically. Attention is focused on the crucial role of dispersive waves for the proper modelling of dynamic strain localisation phenomena, on the effect of the discretisation, on the dispersive properties of the continuum, and on mesh sensitivity in numerical simulations.

\section{Introduction}

By now there exists ample experimental evidence that the residual loadcarrying capacity of cohesive-frictional materials such as sands, clays, rocks and concretes is noticeably below the peak strength. Although the extent to which the values of the peak strength and the residual strength differ depends on the particular material and matters such as confining pressure, a descending branch in the measured load-displacement curve is commonly observed (e.g., Read and Hegemier 1984, van Mier 1984).

There is hardly any doubt that micro-structural changes in the specimen are responsible for this effect. When adopting continuum mechanics as a framework to model this phenomenon additional terms have to be included that represent these changes in the micro-structure. In recent years several approaches have been pursued to achieve this goal. Pijaudier-Cabot and Bažant (1987) have suggested to use a non-local formalism in which the damage variable is averaged over a domain in a weighted sense. Aifantis (1984), Coleman and Hodgdon (1985), Schreyer and Chen (1986), Lasry and Belytschko (1988), Mühlhaus and Aifantis (1991) and de Borst and Mühlhaus (1992) have suggested to enhance the continuum model by incorporating higher-order strain gradients. The introduction of gradient terms can be viewed as an approximation of fully non-local models whereby the non-local 
strain is expanded in a truncated Taylor series (cf. Mühlhaus and Aifantis 1991, de Borst and Mühlhaus 1991), but can also be motivated directly since below a certain size scale the interaction between the micro-structural carriers of the deformation is non-local. In an alternative approach Mühlhaus (1989), Mühlhaus et al. (1991), de Borst (1991a, 1991b, 1992) and de Borst and Sluys (1991) have departed from the standard (Boltzmann) continuum and have used the structure of a Cosserat continuum to introduce microstructure into the field equations. For problems with a high mode- $\Pi$ intensity they have shown this approach to be highly versatile. Finally, it has been emphasised that rate effects cannot be ignored when the loading conditions are transient. Indeed, from a physical point of view the extension of the inelastic constitutive relations with viscous terms is most natural and has been applied to metals (Needleman 1988), to soils (Loret and Prévost 1990, Sluys 1992) and to concrete (Sluys and de Borst 1992).

Apart from physical motivations to include higher-order terms or rate effects in the continuum description there are also compelling reasons from a mathematical point of view. For the formulation of constitutive models the observed descending branch in the load-deflection curve is mapped onto a stress-strain diagram by an affine transformation, that is, stress and strain are computed as the quotients of the force and the virgin load-carrying cross-section, and of the displacement and the length of the specimen, respectively. Clearly, this procedure results in a negative slope of the stressstrain diagram (commonly called strain softening) beyond peak stress level. Under static loading conditions incorporation of such a negative slope in a standard, rate-independent continuum model results in loss of ellipticity of the field equations. Conversely, loss of hyperbolicity occurs when dynamic loading conditions are considered. In both cases well-posedness of the rate boundary value problem is lost and the mathematical model ceases to be a proper description of the physics. In numerical simulations this loss of well-posedness becomes manifest through an extreme mesh sensitivity. Near failure all deformation concentrates in the smallest possible zone that can be resolved by the grid. Upon mesh refinement the failure zone collapses into a discrete plane (localisation) and the energy consumption during the failure process tends to zero.

In this contribution we shall consider two approaches to regularise the field equations in the presence of softening, namely the gradient model and the rate-dependent model. The effect of these regularisation methods is that the width of the localisation zone and the energy consumption during failure remain finite. Attention is focused on dynamic phenomena. By a combi- nation of analytical and numerical techniques it is investigated how waves propagate in a strain-softening gradient-dependent or rate-dependent continuum. It turns out that the role of dispersion, i.e. waves with different wave numbers propagate with different velocities, is of pivotal importance in dynamic localisation phenomena. Although it is known for quite some time that wave propagation in higher-order continua is dispersive - indeed this property has motivated Eringen $(1972,1974)$ to use non-local elasticity for modelling certain wave propagation phenomena - it has been recognised only recently that the fact that in a dispersive medium the shape of a pulse can be altered during propagation is crucial for the formation of shear bands under dynamic loading conditions (de Borst and Sluys 1991, Sluys 1992, Sluys and de Borst 1992).

\section{Wave Propagation in Standard, Rate-independent Softening Plasticity}

Consider an initial value problem in one spatial direction. The governing equations for motion and continuity can then be stated in a rate format as

$$
\frac{\partial \dot{\sigma}}{\partial x}=\rho \frac{\partial^{2} v}{\partial t^{2}}
$$

with $\rho$ the mass density and

$$
\dot{\varepsilon}=\frac{\partial v}{\partial x}
$$

in which velocity $v=\dot{u}$ and $\dot{\sigma}$ the stress rate. For the constitutive relation we shall use a plasticity formalism, so that the strain rate $\dot{\varepsilon}$ is decomposed into an elastic contribution $\dot{\varepsilon}^{e}$ and a plastic contribution $\dot{\varepsilon}^{p}$ :

$$
\dot{\varepsilon}=\dot{\varepsilon}^{e}+\dot{\varepsilon}^{p}
$$

The elastic component is related to the stress rate $\dot{\sigma}$ via a bijective relation

$$
\dot{\sigma}=E \dot{\varepsilon}^{p}
$$

with $E$ Young's modulus. The strain-softening model is assumed to have the following general format: 


$$
\sigma=f\left(\varepsilon^{p}\right)
$$

or in a rate form

$$
\dot{\sigma}=f^{\prime} \dot{\varepsilon}^{p}
$$

where the prime denotes differentiation with respect to the plastic strain $\varepsilon^{p}$. Softening occurs if $f^{\prime}<0$. Combining eqs. (2)-(4) and (6), and differentiating with respect to $x$ yields

$$
\frac{\partial \dot{\sigma}}{\partial x}=\frac{f^{\prime} E}{E+f^{\prime}} \frac{\partial^{2} v}{\partial x^{2}}
$$

Substituting eq. (1) into (7) we obtain the wave equation for a one-dimensional strain-softening bar

$$
\frac{E+f^{\prime}}{c_{e}^{2}} \frac{\partial^{2} v}{\partial t^{2}}-f^{\prime} \frac{\partial^{2} v}{\partial x^{2}}=0
$$

with $c_{e}=\sqrt{E / \rho}$ the linear elastic, longitudinal wave velocity (the so-called bar wave velocity). This second-order partial differential equation is linear if $f^{\prime}$ is constant (linear strain softening) and quasi-linear if $f^{\prime}$ is a function of $\varepsilon^{p}$ (non-linear strain softening). The character of the solution of eq. (8) can be investigated by means of its characteristics, which represent the directions along which the solution develops. We consider the variation of the first derivatives of velocity $v$ with respect to $t$ and $x$

$$
\begin{aligned}
& \mathrm{d}\left(\frac{\partial v}{\partial t}\right)=\frac{\partial^{2} v}{\partial t^{2}} \mathrm{~d} t+\frac{\partial^{2} v}{\partial x \partial t} \mathrm{~d} x \\
& \mathrm{~d}\left(\frac{\partial v}{\partial x}\right)=\frac{\partial^{2} v}{\partial x \partial t} \mathrm{~d} t+\frac{\partial^{2} v}{\partial x^{2}} \mathrm{~d} x
\end{aligned}
$$

Combination of eqs. (9) and (10) with the wave equation for the strainsoftening bar (eq. 8) yields a system of three second-order differential equations. The characteristic determinant reads

$$
D=\left(E+f^{\prime}\right) / c_{e}^{2} \mathrm{~d} x^{2}-f^{\prime} \mathrm{d} t^{2} .
$$

If $D \neq 0$ a unique solution in the $u-x-t$ space can be determined. However, if $D=0$ the system of equations is dependent and a curve in the $u-x-t$ plane coincides with the characteristic directions

$$
\frac{\mathrm{d} x}{\mathrm{~d} t}= \pm c_{e} \sqrt{\frac{f^{\prime}}{E+f^{\prime}}} .
$$

For a wave equation the characteristics $( \pm \mathrm{d} x / \mathrm{d} t)$ coincide with the wave speeds $( \pm c)$. If we have softening $\left(f^{\prime}<0\right)$ the characteristics and therefore the wave speeds will be imaginary and the wave equation loses hyperbolicity. In fact, the domain is split up into an elliptic part, in which waves do not have the ability to propagate (standing waves), and into a hyperbolic part with propagating waves. Spatial interaction between the two domains is impossible. The loss of hyperbolicity means that the rate boundary value problem becomes ill-posed (Benallal et al. 1991) and therefore ceases to be a meaningful description of the physics of the problem.

As alluded to in the introduction, dispersion of waves is of crucial importance for properly modelling dynamic localisation phenomena. Waves are called dispersive if harmonic waves with a different frequency propagate with different phase velocities. Denoting $\omega$ as the angular frequency and $k$ as the wave number counting the number of wave lengths $\lambda$ in the bar over $2 \pi$

$$
k=\frac{2 \pi}{\lambda}
$$

this means that the phase velocity $c=\omega / k$ must be a function of wave number (Whitham 1974). A travelling wave is composed of harmonic waves which have different phase velocities and therefore its shape is altered during propagation.

For a dispersion analysis we consider a single linear harmonic wave which propagates through a one-dimensional continuum

$$
v(x, t)=a e^{i(k x-\omega t)},
$$

A dispersion relation can be obtained if eq. (14) is substituted into the wave equation (8), which yields

$$
\omega=c_{e} \sqrt{\frac{f^{\prime}}{E+f^{\prime}}} k .
$$


For the classical strain-softening bar considered here the phase velocity $c$ is clearly independent of the wave number $k$, so that the waves are nondispersive. Accordingly the shape of an arbitrary loading wave cannot be transformed into a stationary wave.

To investigate the consequences of the mathematical statements of ill-posedness and imaginary wave speeds, we shall now derive the analytical solution of a bar of length $L$ which is fixed at one side and loaded by a dynamic tensile force at the other side (Sluys (1992), see also Bažant and Belytschko (1985), who found an exact analytical solution for a one-dimensional strainsoftening bar with prescribed velocity at both sides of the bar). This longitudinal wave propagation problem is sketched in Figure 1. We use a linear strain-softening model $\left(f^{\prime}=h=\right.$ constant $)$ and a step load $\left(t_{d}=0\right)$. Starting at the right boundary the transient wave propagates through the bar and reflects at the left boundary. If $f_{t} / 2<q_{0}<f_{t}$, with $f_{t}$ the tensile strength, the tensile strength is exceeded after reflection. The strain-softening branch is entered and a localised softening zone of a width $w$ emerges. Since the wave equation now becomes elliptic, interaction over finite distances is immediate, the localisation zone cannot extend and remains infinitely small $(w \rightarrow 0)$. A solution to the initial value problem is (note that there may exist other solutions, cf. Sluys 1992):

$u(x, t)=\frac{H\left(t-\frac{L-x}{c_{e}}\right)}{\rho c_{e}} q_{0}\left(t-\frac{L-x}{c_{e}}\right)+\frac{H\left(t-\frac{L+x}{c_{e}}\right)}{\rho c_{e}} q_{0}\left(t-\frac{L+x}{c_{e}}\right)$,

in which $H$ is the Heaviside step function,

$$
\varepsilon(x, t)=\frac{q_{0}}{E}\left[H\left(t-\frac{L-x}{c_{e}}\right)-H\left(t-\frac{L+x}{c_{e}}\right)\right]+\frac{2 q_{0}}{\rho c_{e}}\left(t-\frac{L}{c_{e}}\right) \delta(x)
$$

with $\delta(x)$ the Dirac delta function, and

$$
\sigma(x, t)=q_{0}\left[H\left(t-\frac{L-x}{c_{e}}\right)-H\left(t-\frac{L+x}{c_{e}}\right)\right] .
$$

If stresses and strains are known the energy consumption in the bar can be calculated as
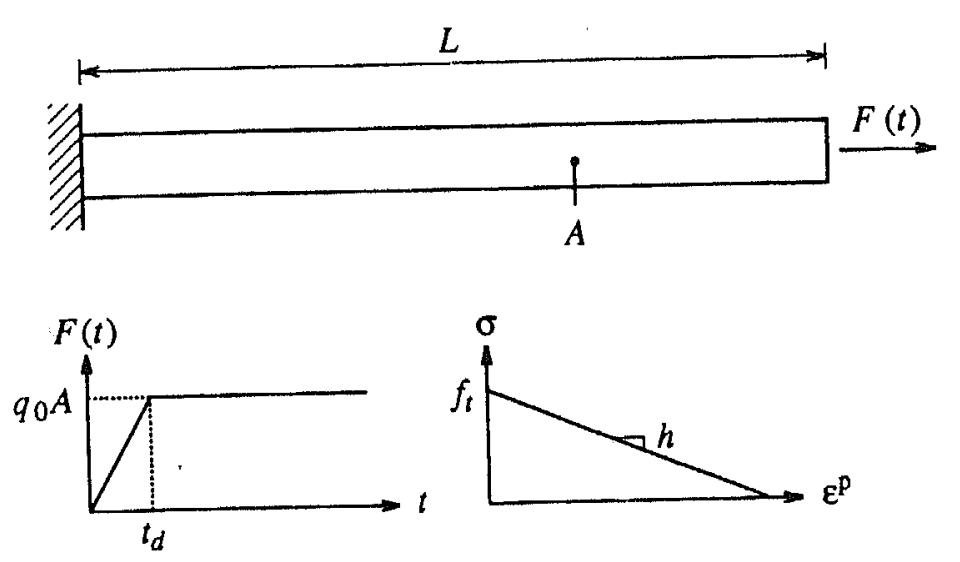

$$
\begin{array}{lll}
\text { geometry : } & \text { loading: } & \text { material data: } \\
L=100 \mathrm{~mm} & q_{0}=0.75 f_{t} & E=20000 \mathrm{~N} / \mathrm{mm}^{2} \\
A=1 \mathrm{~mm}^{2} & t_{d}=0.0 \mathrm{~s} & \rho=2 \cdot 10^{-8} \mathrm{Ns}^{2} / \mathrm{mm}^{4} \\
& & f_{t}=2 \mathrm{~N} / \mathrm{mm}^{2} \\
& & h=-1000 \mathrm{~N} / \mathrm{mm}^{2}
\end{array}
$$

FIGURE 1. One-dimensional bar problem in tension.

$$
U(t)=\frac{q_{0}^{2} A c_{e}}{2 E}\left[t-2\left(t-\frac{L}{c_{e}}\right) H\left(t-\frac{L}{c_{e}}\right)\right] \quad \text { for } \quad 0<t<2 \frac{L}{c_{e}}
$$

According to this solution the strain tends to infinity after reflection in a localisation zone of a zero width. In fact, the solution of the elliptic equation is a standing wave, described by a Dirac delta function, which does not have the ability to extend. The stress drops to zero instantly and the wave reflects on the softening zone as on a free boundary. Spatial interaction between the elliptic and the hyperbolic system is not possible. The tensile wave returns as a pressure wave instead of a superposition of tensile waves which would happen for reflection of an elastic wave on a fixed boundary. Finally, eq. (19) shows that after reflection from $t=L / c_{e}$ the bar is unable to consume inelastic energy and the elastic energy of the reflected wave is the total energy in the bar.

The problem of a uniaxial bar under impact loading will now be investigated 
numerically. The geometry, loading and material data are represented in Figure 1. Use of these parameters yields a linear elastic wave speed $c_{e}=$ $1000 \mathrm{~m} / \mathrm{s}$. We consider a block wave with a vertical stress front which corresponds to $t_{d}=0$. The time integration of the field equations has been done with the Newmark scheme $(\beta=1 / 4, \gamma=1 / 2)$. For the time step we take $\Delta t=5 \cdot 10^{-7} \mathrm{~s}$. Use has been made of quadratic elements with a threepoint Gauss integration scheme. A consistent mass matrix has been used for all analyses with the standard continuum model. The bar is divided into $10,20,40$ and 80 elements, respectively.

The response of the bar is linearly elastic until the loading wave reaches the left boundary. The doubling in stress $\left(2 q_{0}=\frac{3}{2} f_{t}\right)$ due to reflection of the tensile wave marks the onset of softening. A localisation zone of intense straining subsequently emerges.

In Figure 2 the displacements and the strains for the different meshes are plotted at $t=\frac{3}{2} \frac{L}{c_{e}}=0.15 \cdot 10^{-3} \mathrm{~s}$, that is when the wave has reflected at the left boundary and has returned to $x=L / 2$. Note that the result for the discretisation with 80 elements has not been plotted because at $t=0.15 \cdot 10^{-3} \mathrm{~s}$ the bar has already failed. Mesh sensitivity is obvious: strain localisation occurs in the form of a jump in displacements in only one integration point. This is the smallest possible zone which is in agreement with the analytical solution eqs. (16)-(19).

The stress profiles after reflection as plotted in Figure 3 show that the amount of wave reflection also depends on the mesh: for more elements there is a larger reduction in stress of the reflected wave. As soon as the stress has become zero one integration point starts to act as a free boundary on which the tensile wave reflects as a pressure wave. Summation of a tensile wave propagating to the left and a pressure wave propagating to the right yields a zero stress situation. Finally, the development of the consumption of the energy $U$ in the bar depends on the number of elements in the mesh, Figure 3 . In the limiting case of an infinite number of elements failure occurs at $t=L / c_{e}$ without further energy consumption in the strain-softening zone of the bar. The stress drops to zero instantly and the wave reflects as a pressure wave. The elastic energy gradually vanishes in the bar with the returning pressure wave.
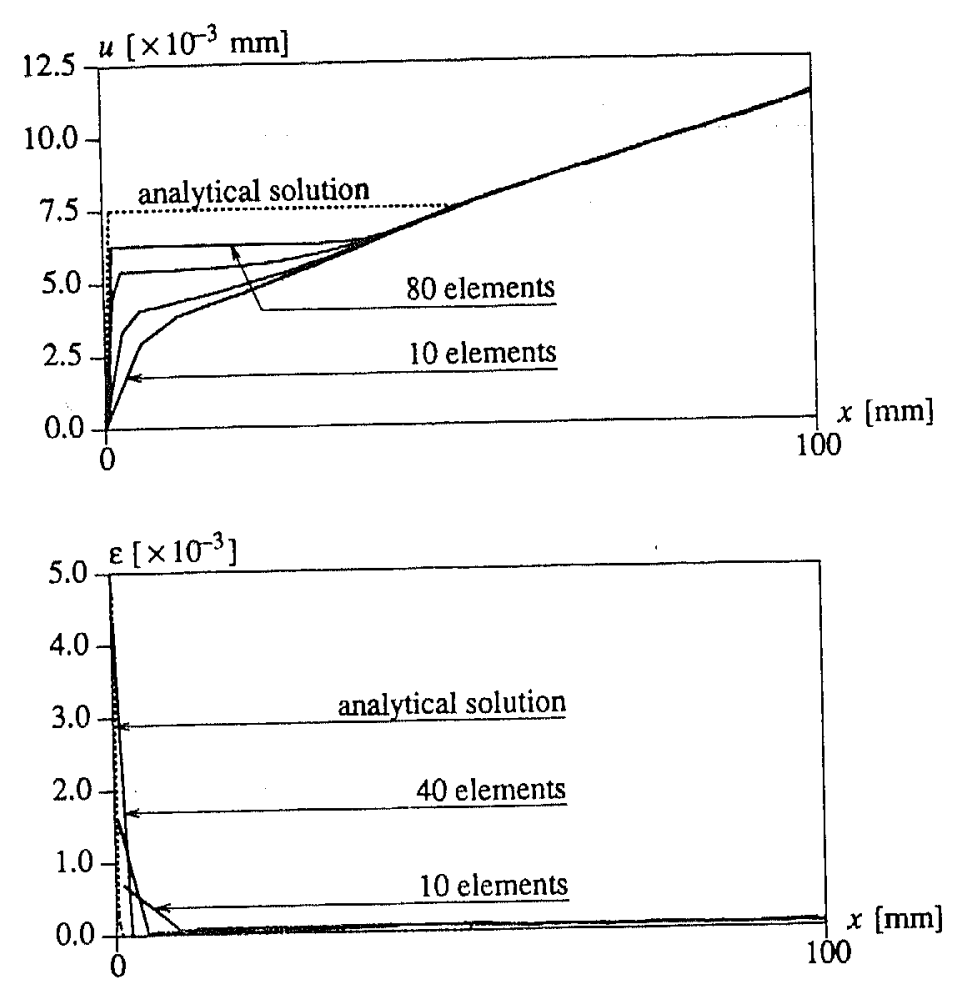

FIGURE 2. Mesh-dependent results with a standard strain-softening model. Top: Displacements along the bar at $t=\frac{3}{2} \frac{L}{c_{e}}=0.15 \cdot 10^{-3} \mathrm{~s}$. Bottom: Strain localisation along the bar at $t=\frac{3}{2} \frac{L}{c_{e}}=0.15 \cdot 10^{-3} \mathrm{~s}$.

\section{Dispersive Waves in Gradient-dependent Softening Plasticity}

\subsection{MODEL FORMULATION}

In the present gradient plasticity theory the yield function is assumed to depend not only upon the plastic strain $\varepsilon^{p}$, but also upon its second derivative. While the equation of motion (1), the kinematic equation (2) and the constitutive equations (3)-(4) remain unchanged, the strain-softening part now reads

$$
\sigma=f\left(\varepsilon^{p}, \partial^{2} \varepsilon^{p} / \partial x^{2}\right)
$$



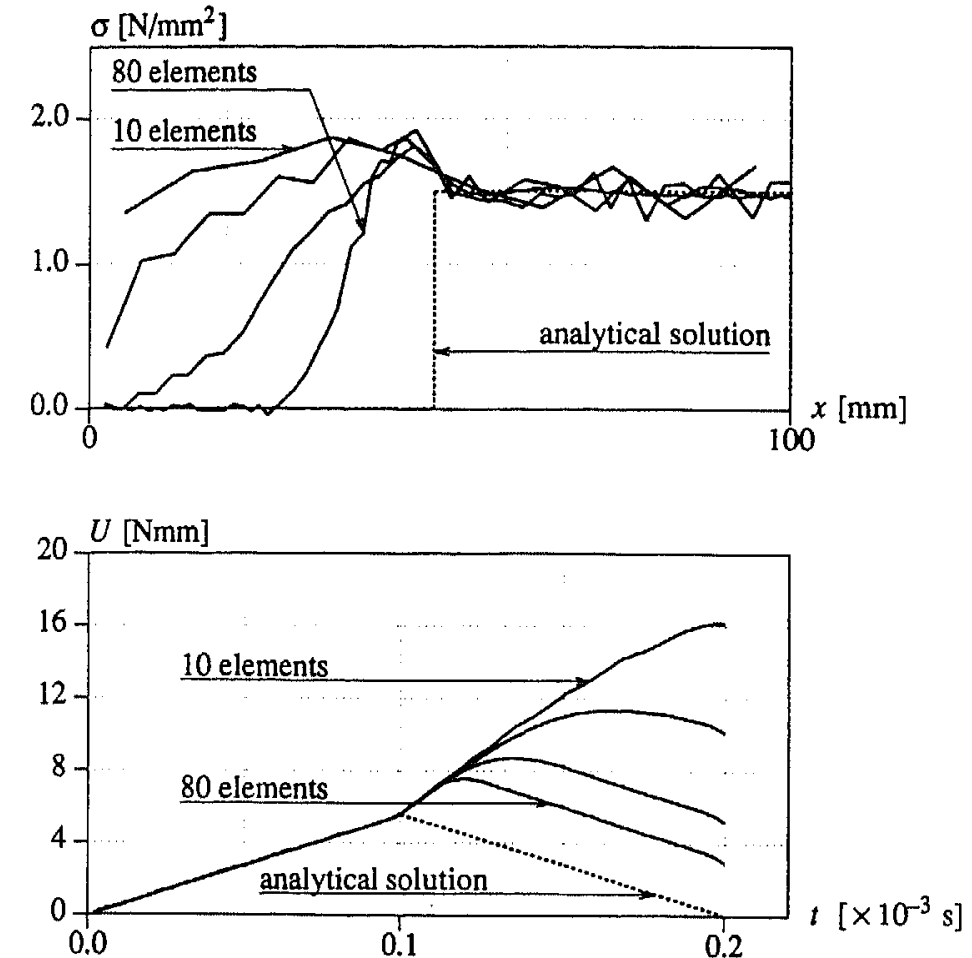

FIGURE 3. Mesh-dependent results with a standard strain-softening model. Top: Stress profiles along the bar at $t=\frac{3}{2} \frac{L}{c_{e}}=0.15 \cdot 10^{-3} \mathrm{~s}$. Bottom: Energy consumption of the bar.

In case of linear softening ( $f^{\prime}=h=$ constant), the rate formulation becomes

$$
\dot{\sigma}=h \dot{\varepsilon}^{p}-\bar{c} \frac{\partial^{2} \dot{\varepsilon}^{p}}{\partial x^{2}}
$$

where in the analyses presented here,

$$
\bar{c}=-\frac{\partial f}{\partial\left(\partial^{2} \varepsilon^{p} / \partial x^{2}\right)}
$$

has also been assumed to be a constant. A problem with the application of standard numerical (finite element) computations for elasto-plastic solids is that eq. (21) is a partial differential equation. To solve the rate boundary value problem numerically Mühlhaus and Aifantis (1991) and de Borst and Mühlhaus (1992) have proposed to consider the inelastic strain rate $\dot{\varepsilon}^{p}$ as an independent unknown in addition to the axial velocity $v$. For this purpose we substitute the decomposition of strain rates (3) and the elastic part of the stress-strain law (4) in the weak form of the equation of motion (1) and in the softening function in rate format (21). Using the divergence theorem and neglecting boundary tractions this gives

$$
\int_{V} \delta v \rho \frac{\partial^{2} v}{\partial t^{2}} \mathrm{~d} V+\int_{V} \delta \dot{\varepsilon} E\left(\dot{\varepsilon}-\dot{\varepsilon}^{p}\right) \mathrm{d} V=0
$$

and

$$
\int_{V} \delta \dot{\varepsilon}^{p}\left[E\left(\dot{\varepsilon}-\varepsilon^{p}\right)-h \dot{\varepsilon}^{p}+\bar{c} \frac{\partial^{2} \varepsilon^{p}}{\partial x^{2}}\right] \mathrm{d} V=0
$$

which will be used as a starting point of the finite element discretisation in section 3.3 .

\subsection{DISPERSION ANALYSIS}

To analyse wave propagation in a gradient-dependent one-dimensional eleTo analyse wave propagation in a gradient-dependent
ment we combine the constitutive equation (21) with eqs. (2)-(4) and differentiate the result with respect to $x$

$$
\frac{\partial}{\partial x}\left(-\frac{\bar{c}}{E} \frac{\partial^{2} \dot{\sigma}}{\partial x^{2}}+\frac{E+h}{E} \dot{\sigma}\right)=\frac{\partial^{2}}{\partial x^{2}}\left(h v-\bar{c} \frac{\partial^{2} v}{\partial x^{2}}\right) .
$$

If we combine this result with the equation of motion (1) we obtain a fourthorder differential equation for the one-dimensional gradient-dependent, strain-softening bar

$$
\bar{c}\left(\frac{\partial^{4} v}{\partial x^{4}}-\frac{1}{c_{e}^{2}} \frac{\partial^{4} v}{\partial x^{2} \partial t^{2}}\right)+\frac{E+h}{c_{e}^{2}} \frac{\partial^{2} v}{\partial t^{2}}-h \frac{\partial^{2} v}{\partial x^{2}}=0 .
$$

Note that if $\bar{c} \rightarrow 0$ the wave equation for the classical strain-softening bar (eq. 8) is recovered with imaginary characteristics and imaginary wave speeds.

The condition of eq. (26) can be investigated by means of its characteristics. To this end we consider the variation of two third-order derivative terms of $v$ 


$$
\begin{gathered}
\mathrm{d}\left(\frac{\partial^{3} v}{\partial x^{3}}\right)=\frac{\partial^{4} v}{\partial x^{3} \partial t} \mathrm{~d} t+\frac{\partial^{4} v}{\partial x^{4}} \mathrm{~d} x \\
\mathrm{~d}\left(\frac{\partial^{3} v}{\partial x^{2} \partial t}\right)=\frac{\partial^{4} v}{\partial x^{2} \partial t^{2}} \mathrm{~d} t+\frac{\partial^{4} v}{\partial x^{3} \partial t} \mathrm{~d} x
\end{gathered}
$$

Combination of eq. (27) and (28) and the wave equation for the gradientdependent bar, eq. (26), yields a system of three fourth-order differential equations with a characteristic determinant

$$
D=\bar{c}\left[\mathrm{~d} t^{2}-\left(1 / c_{e}^{2}\right) \mathrm{d} x^{2}\right] .
$$

With $D=0$ the characteristics are equal to the elastic bar velocity $\pm c_{e}$ and remain real when strain softening occurs. So, the wave equation remains hyperbolic and the initial value problem is well-posed.

We now carry out a dispersion analysis for the gradient-dependent bar. Substitution of the general solution (14) for a single harmonic wave into the wave equation (26) gives the dispersion relation for the gradient-dependent bar

$$
\bar{c} k^{4}-\bar{c} / c_{e}^{2} k^{2} \omega^{2}-(E+h) / c_{e}^{2} \omega^{2}+h k^{2}=0
$$

Considering the positive root for $\omega$

$$
\omega=c_{e} \sqrt{\frac{h+\bar{c} k^{2}}{E+h+\bar{c} k^{2}}} \quad k
$$

it becomes clear that the classical non-dispersive relation (15) is recovered when $\bar{c} \rightarrow 0$. For $\bar{c} \neq 0$ and in case of the one-dimensional bar problem the dispersion relation is plotted in Figure 4. The phase velocity $c=\omega / k$ of the harmonic wave reads

$$
c=c_{e} \sqrt{\frac{h+\bar{c} k^{2}}{E+h+\bar{c} k^{2}}}
$$

which relation is depicted graphically in Figure 5. The phase speed $c$ depends on the wave number $k$ and, consequently, wave propagation is dispersive for the gradient-dependent bar (Whitham 1974). Owing to the fact that different harmonic waves propagate with different velocities the shape of a pulse is altered and, in contrast to the standard continuum model, a loading wave can be transformed into a stationary localisation wave. For a gradient-dependent model the phase speed therefore not necessarily becomes imaginary at the onset of softening as in a standard continuum model. From eq. (32) it follows that the phase velocity remains real if

$$
k \geq \sqrt{-\frac{h}{\bar{c}}} \quad \text { and thus } \quad \lambda \leq 2 \pi l, \quad \text { with } \quad l=\sqrt{-\frac{\bar{c}}{h}} .
$$

The parameter $l$ is the internal length scale in the gradient-dependent model. If $k\left\langle l^{-1}\right.$ or wave length $\lambda>2 \pi l$ we recover the situation in which a disturbance $\delta v$ is unbounded and stability in the sense of Lyapunov is lost (i.e. a small disturbance of boundary data results in large changes of the response). However, strain-softening regions remain small and no wave lengths larger than $2 \pi l$ can occur because they do not fit within the strainsoftening region. Consequently, all phase velocities remain real because the first-order wave with the lowest wave number (largest wave length) has a wave number which is larger than the critical value in eq. (33). In the numerical analyses we will observe that all higher frequencies which are present in a loading wave vanish under the influence of nonlinear material behaviour and we obtain a stationary harmonic localisation wave with a width equal to the maximum wave length $w=\lambda=2 \pi l$.

\subsection{THE INFLUENGE OF THE DISCRETISATION ON DISPERSIVE WAVES}

The dispersion analysis carried out for the one-dimensional bar can also be done for a bar discretised in the spatial dimension. By using a representation in finite elements the interaction between the physical and numerical dispersion can be determined. The size of the finite elements and the mass distribution within the element cause a contribution to the dispersion phenomenon. Since we use the implicit Newmark time integration scheme with $\beta=1 / 4$ and $\gamma=1 / 2$ the small contribution of the time integrator to numerical dispersion is neglected. An analysis of the discretisation influence offers the possibility to derive a condition for the minimum number of finite elements needed in the localisation zone for an accurate representation. The influence of the discretisation on dispersion behaviour has been discussed before by Huerta and Pijaudier-Cabot (1992). 


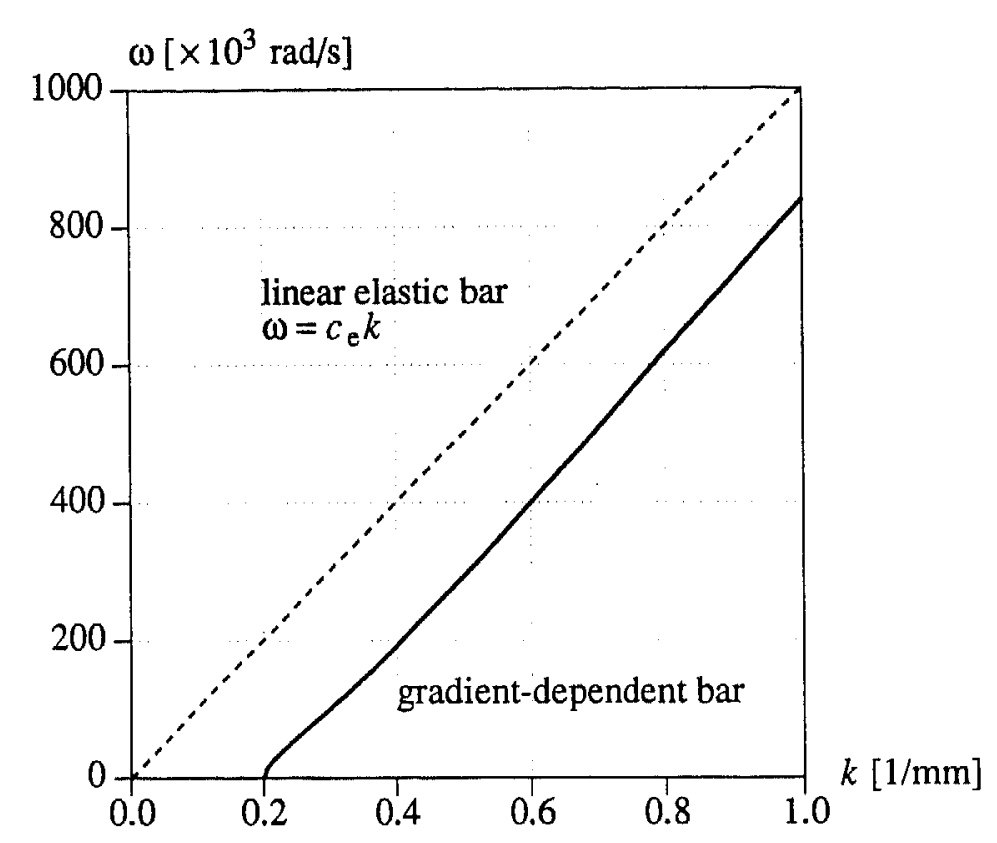

FIGURE 4. Dispersion relation for the gradient-dependent bar.

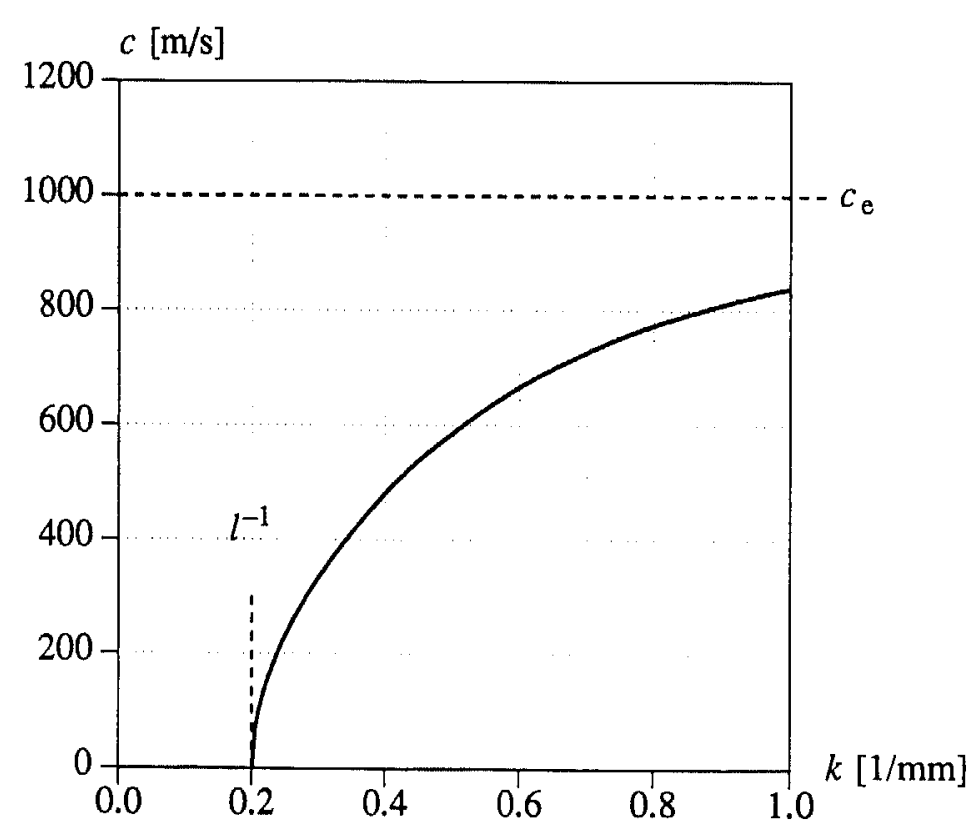

FIGURE 5. Phase velocity - wave number curve for gradient-dependent model.
For the gradient model we assume a finite element representation of the weak form of eqs. (23) and (24) via

$$
\begin{gathered}
v(x)=\mathbf{H a}, \\
\dot{\varepsilon}(x)=\mathbf{B a}, \\
\dot{\varepsilon}^{p}(x)=\mathbf{h}^{T} \boldsymbol{\Xi}, \\
\frac{\partial^{2} \dot{\varepsilon}^{p}(x)}{\partial x^{2}}=\mathbf{p}^{T} \boldsymbol{\Xi},
\end{gathered}
$$

in which a denotes the nodal velocities and $\boldsymbol{\Xi}$ denotes the nodal plastic strain rates. Matrix $\mathbf{H}$ contains the interpolation polynomials for the displacement field and $\mathbf{B}=\mathbf{L H}$, in which $\mathbf{L}$ is the differential operator matrix. An important issue is now the order of interpolation of the variables $v$ and $\dot{\varepsilon}^{p}$. While $C^{0}$-interpolants suffice for $v$, the presence of a second spatial derivative of $\dot{\varepsilon}^{p}$ requires $C^{1}$-continuous shape functions. In the numerical analyses presented here, Hermitian functions have been used for $\mathbf{h}$ for this purpose. If we take a bar element with an element size $d$ and node numbers $j$ and $j+1$ we obtain

$\dot{\varepsilon}^{p}(x)=\left[1-\frac{3 x^{2}}{d^{2}}+\frac{2 x^{3}}{d^{3}}, x-\frac{2 x^{2}}{d}-\frac{x^{3}}{d^{2}}, \frac{3 x^{2}}{d^{2}}-\frac{2 x^{3}}{d^{3}},-\frac{x^{2}}{d}+\frac{x^{3}}{d^{2}}\right]\left[\begin{array}{c}\Xi_{j} \\ \mathrm{~d} \Xi_{j} \\ \Xi_{j+1} \\ \mathrm{~d} \Xi_{j+1}\end{array}\right]$

and $\mathbf{p}$ is calculated by differentiating the polynomials of $\mathbf{h}$ twice. Substitution of eqs. (34)-(37) in eqs. (23) and (24) yields

$$
\begin{aligned}
& \int_{V} \rho \mathbf{H}^{T} \mathbf{H} \mathrm{d} V \ddot{\mathbf{a}}+\int_{V} E \mathbf{B}^{\mathbf{T}} \mathbf{B} \mathrm{d} V \mathbf{a}-\int_{V} E \mathbf{B}^{T} \mathbf{h}^{T} \mathrm{~d} V \boldsymbol{\Xi}=0 \\
& \int_{V} E \mathbf{h} \mathbf{B} \mathrm{d} V \mathbf{a}-\int_{V}(h+E) \mathbf{h} \mathbf{h}^{T} \mathrm{~d} V \boldsymbol{\Xi}+\int_{V} \bar{c} \mathbf{h} \mathbf{p}^{T} \mathrm{~d} V \boldsymbol{\Xi}=0
\end{aligned}
$$

The matrices in eqs. (39) and (40) have been determined for a bar element integrated by a two point quadrature. We assume a mesh with elements of constant length and consider eqs. (39) and (40) for node $j$ 


$$
\begin{aligned}
& \quad \frac{M_{d}}{E}+\frac{1}{d}\left(-a_{j-1}+2 a_{j}-a_{j+1}\right) \\
& -\left(\frac{1}{2} \Xi_{j-1}+\frac{3 d}{32} \mathrm{~d} \Xi_{j-1}-\frac{6 d}{32} \mathrm{~d} \Xi_{j}-\frac{1}{2} \Xi_{j+1}+\frac{3 d}{32} \mathrm{~d} \Xi_{j+1}\right)=0 \\
& \frac{E}{2}\left(-a_{j-1}+a_{j+1}\right) \\
& -\frac{(h+E) d}{4096}\left(540 \xi_{j-1}+126 d \mathrm{~d} \Xi_{j-1}+3016 \Xi_{j}+540 \Xi_{j+1}-126 d \mathrm{~d} \Xi_{j+1}\right) \\
& +\frac{\bar{c}}{128}\left(\frac{132}{d} \Xi_{j-1}+2 \mathrm{~d} \Xi_{j-1}-\frac{264}{d} \Xi_{j}+\frac{132}{d} \Xi_{j+1}-2 \mathrm{~d} \Xi_{j+1}\right)=0 \\
& \quad \frac{E d}{32}\left(3 a_{j-1}-6 a_{j}+3 a_{j+1}\right) \\
& -\frac{(h+E) d}{4096}\left(-126 d \Xi_{j-1}-27 d^{2} \mathrm{~d} \Xi_{j-1}+90 d^{2} \mathrm{~d} \Xi_{j}+\right. \\
& \left.+126 d \Xi_{j+1}-27 d^{2} \mathrm{~d} \Xi_{j+1}\right) \\
& +\frac{\bar{c}}{128}\left(-18 \Xi_{j-1}+3 d \mathrm{~d} \Xi_{j-1}-42 d \mathrm{~d} \Xi_{j}+18 \Xi_{j+1}+3 d \mathrm{~d} \Xi_{j+1}\right)=0 .
\end{aligned}
$$

The parameter $M_{d}$ is dependent on the mass distribution in the finite elements. For a consistent, a lumped and a higher-order mass matrix, respectively, we derive

$$
\begin{aligned}
& M_{d}=\frac{\rho d}{16}\left(3 \frac{\partial^{2} a_{j-1}}{\partial t^{2}}+10 \frac{\partial^{2} a_{j}}{\partial t^{2}}+3 \frac{\partial^{2} a_{j-1}}{\partial t^{2}}\right) \\
& M_{d}=\rho d \frac{\partial^{2} a_{j}}{\partial t^{2}} \\
& M_{d}=\frac{\rho d}{32}\left(3 \frac{\partial^{2} a_{j-1}}{\partial t^{2}}+26 \frac{\partial^{2} a_{j}}{\partial t^{2}}+3 \frac{\partial^{2} a_{j-1}}{\partial t^{2}}\right) .
\end{aligned}
$$

The higher-order mass matrix is obtained by averaging the lumped and the consistent mass matrices.

Similar to the harmonic wave in eq. (14) for the dispersion analysis of the discretised bar we take a consistent set of harmonic solutions

$$
\begin{gathered}
a_{j}=A e^{i(k x-\omega t)}, \\
\Xi_{j}=i k B e^{i(k x-\omega t)}, \\
\mathrm{d} \Xi_{j}=k^{2} C e^{i(k x-\omega t)} .
\end{gathered}
$$

The solution at the neighbouring nodes for the velocity field is then

$$
\begin{aligned}
& a_{j-1}=(\cos k d-i \sin k d) A e^{i(k x-\omega t)}, \\
& a_{j+1}=(\cos k d+i \sin k d) A e^{i(k x-\omega t)} .
\end{aligned}
$$

Substitution of the complete solution (eqs. 47-51) in eqs. (41)-(43) gives a system of three equations for which a non-trivial solution results in a dispersion relation in which the frequency $\omega$ is a function of the wave number $k$ and the finite element size $d$. The dispersion curve of the discretised bar for a consistent mass distribution is plotted in Figure 6 for different sizes $d$ of the finite elements. We observe that refinement of the mesh $(d \rightarrow 0)$ leads to convergence of the dispersion curve to the continuum dispersion curve of Figure 4 . The same tendency is observed in Figure 7 if we plot the phase velocity $c$ against wave number $k$ for different values of $d$. A second observation is that in Figure 7 the point that represents the stationary localisation wave $(c=0)$ gradually moves to a smaller value of $k$ when larger elements are used. This means that the wave length $(=2 \pi / k)$ which represents the width of the localisation zone increases. This is exactly what is observed in the numerical calculations of section 3.4. This widening of the localisation band can be quantified as will be demonstrated next.

Furthermore, it is observed in the Figures 6 and 7 that the deviation between the "discretised" dispersion curve and the "continuum" dispersion curve increases for higher frequencies (and smaller wave lengths). This is in fact a trivial result because the accuracy of the finite element solution rapidly decreases when the wave length is of the same order as the element size. For instance in Figure 6 for $d=4.0 \mathrm{~mm}$ a maximum in the dispersion curve is observed which corresponds to a wave length $\lambda=2 d$. When wave lengths are smaller than $2 d$ this linear element cannot produce accurate results.

The influence of the mass discretisation is shown in Figure 8, in which a consistent, a lumped and a higher-order mass matrix have been used for an 
element size $d=2.0 \mathrm{~mm}$. We observe that a consistent mass matrix provides an upper bound of the continuum value while a lumped mass matrix results in a lower bound value. The higher-order mass matrix gives the most accurate description, even for very high frequencies. The results with respect to mass discretisation are similar to the outcome of accuracy analyses carried out for elastic media (Hughes 1987). A second result of the variation of mass matrices is that the type of distribution does not affect the width of a stationary localisation zone $(c=0)$. This result is logical since inertia effects do not play a role in a stationary localisation zone.

Finally, if we take $c=0$ in the dispersion relation a dependence can be derived between the width of the localisation zone in the discretised continuum $w_{d i s c r}$ and the element size $d$. This result is plotted in Figure 9, in which $w_{d i s c r}$ is normalised with respect to the exact width of the localisation zone $w_{\text {exact }}$. A criterion for the required number of finite elements $n_{\text {elem }}$ in the localisation zone can be derived. Namely, if a $10 \%$ mismatch between discretised and exact value is accepted it follows that

$$
n_{e l e m} \geq \frac{w_{e x a c t}}{d_{c r i \pm 10 \%}}=11.8 .
$$

However, it is noted that the use of elements with a quadratic interpolation for the velocity field results in a much less severe condition.

\subsection{ONE-DIMENSIONAL FINITE ELEMENT ANALYSES}

To further investigate the dispersive character of the gradient-dependent model and the performance with respect to mesh refinement numerical analyses have been carried out for $20,40,80$ and 160 elements, respectively. The calculations have again been carried out with the Newmark time integration scheme with the same constants and time steps as for the standard continuum model. Unless stated otherwise a consistent mass matrix has been used.

Firstly, we have investigated the gradient model by means of the onedimensional bar problem in pure tension (Figure 1). A slight modification has been made to the parameter set by taking a steeper softening branch compared with the classical case: $h=-2000 \mathrm{~N} / \mathrm{mm}^{2}$. This does not necessarily increase the brittleness of the material because the gradient effect "carries" a part of the load. We will observe that the same happens in ratedependent models because of the viscous effect. The extra gradient constant $\bar{c}=50000 \mathrm{~N}$. The values for $h$ and $\bar{c}$ imply an internal length scale para-

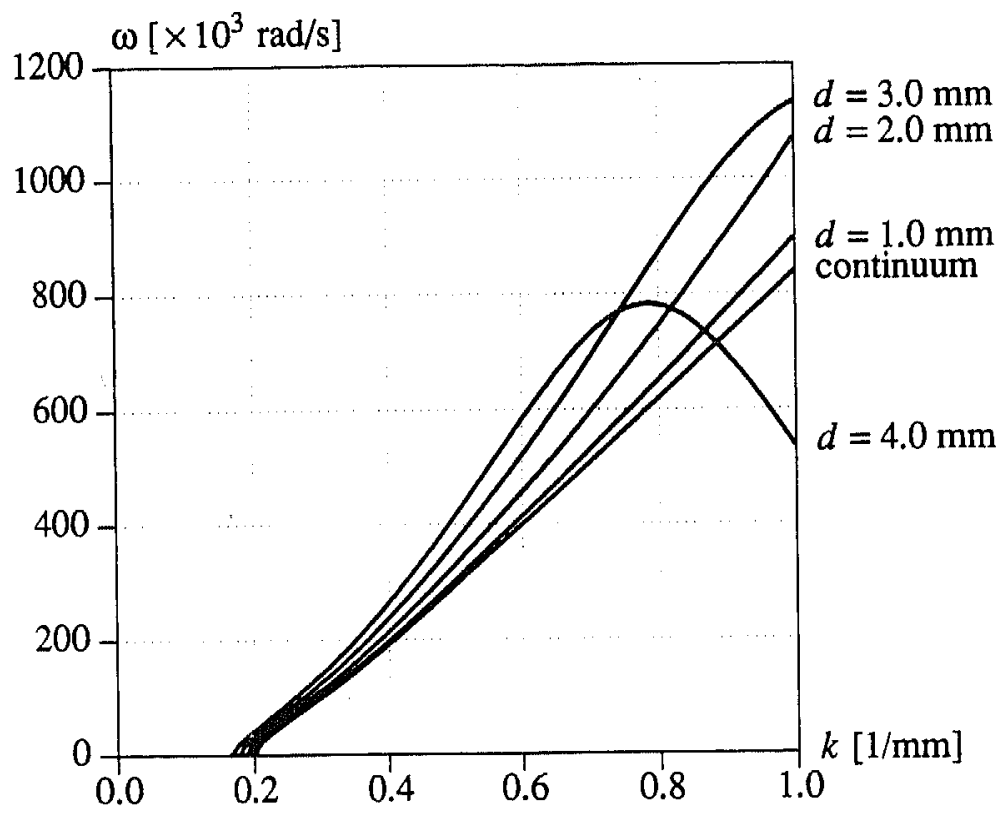

FIGURE 6. Discretised dispersion relation for the gradient-dependent bar.

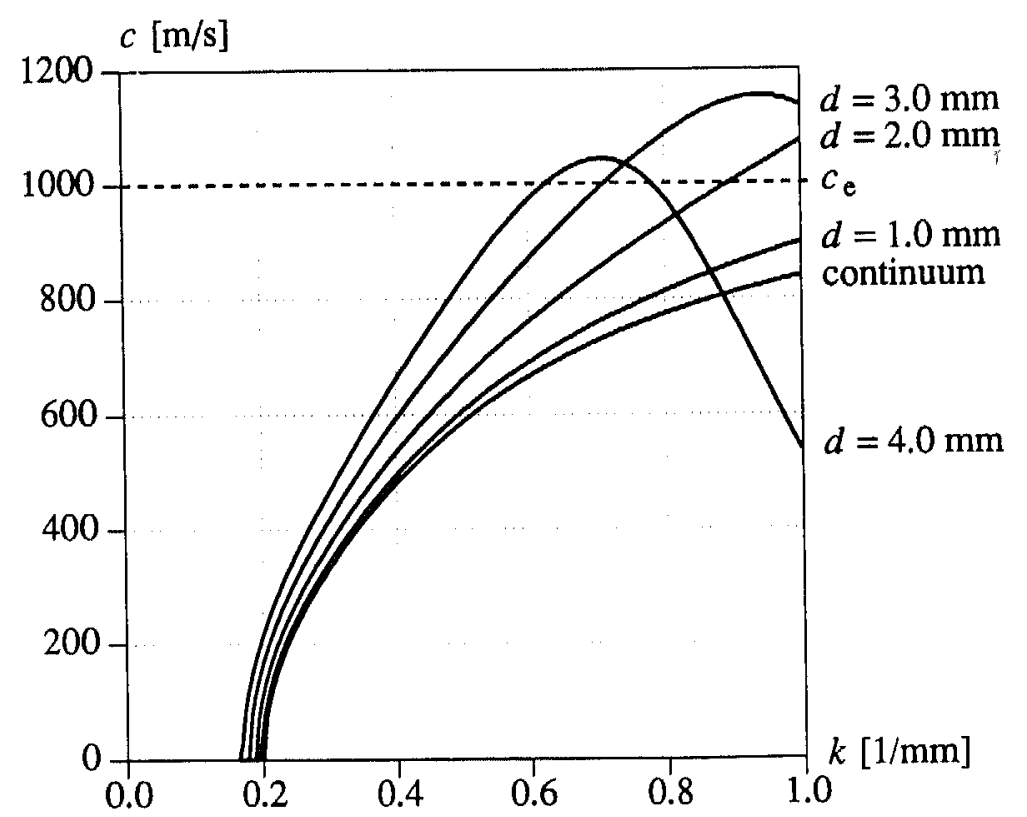

FIGURE 7. Discretised phase velocity - wave number curve for the gradient-dependent bar with a consistent mass matrix. 
$c[\mathrm{~m} / \mathrm{s}]$

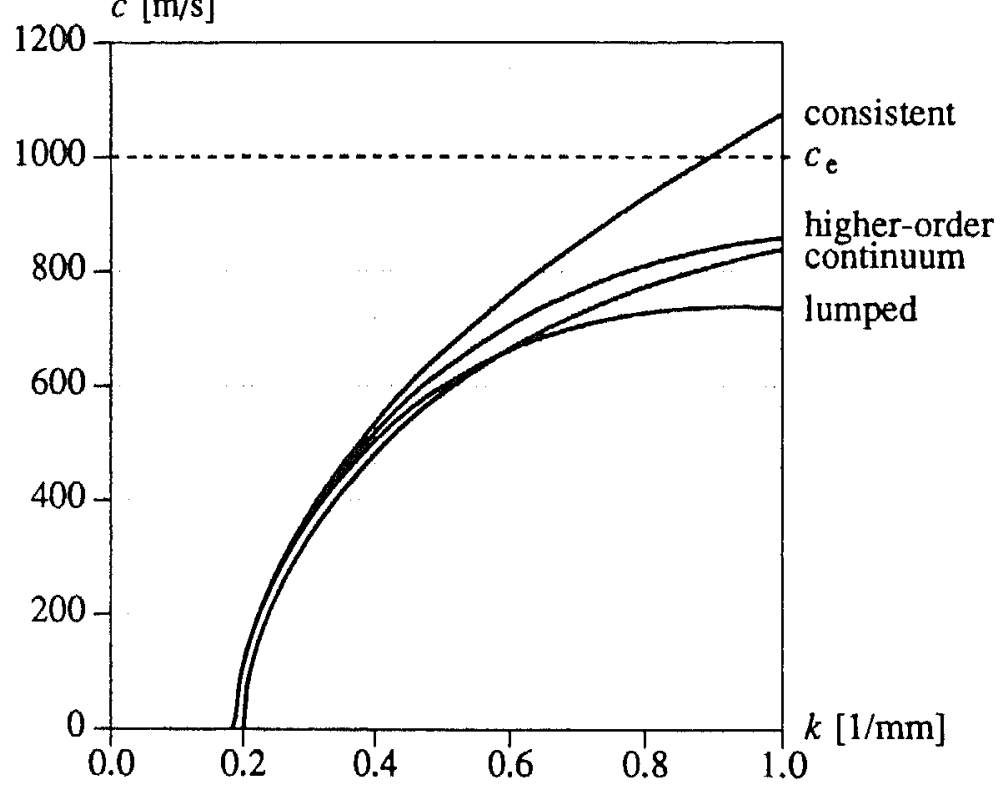

FIGURE 8. Discretised phase velocity - wave number curve for different mass discretisations.

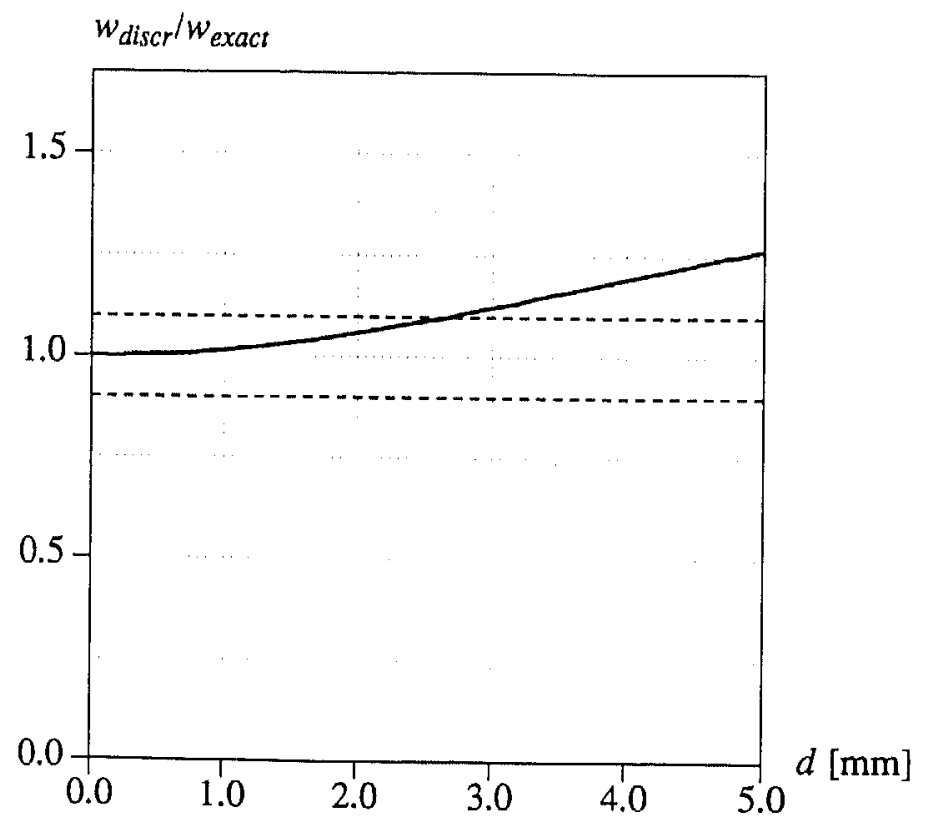

FIGURE 9. Discretisation influence on the localisation band width. meter $l=5 \mathrm{~mm}$ (eq. 33). Again, we consider a block wave $\left(t_{d}=0\right)$ which propagates in a linear-elastic fashion through the bar until reflection occurs and the localisation process is initiated. Extra boundary conditions (cf. de Borst, and Mühlhaus 1992, Sluys 1992) have been applied $\left(\partial \dot{\varepsilon}^{p} / \partial x=0\right)$ at both sides of the bar.

For the gradient model a localisation zone emerges with a width that converges to a finite, constant value upon mesh refinement. In Figure 10 the strain profile is plotted for different meshes. The coarser meshes with 20 and 40 elements still deviate somewhat but the fine meshes give almost identical results. In the same figure the development of the localisation band has been plotted at several time steps. First, the width of the zone increases after reflection but later the speed of extension of the zone vanishes and a localisation band of constant width arises $(w / 2 \approx 16 \mathrm{~mm})$. Owing to dispersion and material damping the higher-order waves are attenuated and the shape of the loading wave changes into a first-order harmonic wave with velocity $c$ equal to zero. This corresponds to a wave number $k=0.21 / \mathrm{mm}$ and a harmonic wave length $\lambda=2 \pi l=31.4 \mathrm{~mm}$, eq. (33). So, the numerical localisation band width $w$ equals the first order wave length $\lambda$ belonging to a phase velocity $c=0$ under the condition that the localisation band has developed completely. According to condition (52), which came out of the discrete analysis of dispersive waves, at least 12 elements are needed in the localisation zone, which corresponds to a total number of $(100 / 15.7) \cdot 6 \approx 38$ elements for a $10 \%$ error in the width of the band. This is in good agreement with the results in Figure 10, from which we observe that only the 20 element mesh gives an error that is larger than $10 \%$. In Figure 11 it is shown that mesh insensitivity is not only obtained for the width of the localisation zone but also for the wave reflection patterns and the energy consumption. The stress profiles (Figure 11 - top) are a superposition of the loading wave travelling to the left and the reflected wave travelling to the right. The patterns are more or less identical for the four meshes and it appears that wave reflection in a gradient-dependent bar is not determined by the number of elements. For the gradient model the localisation zone thus converges to a non-zero width and to physically realistic responses for the wave reflection on and the energy consumption in the zone.

The internal length scale parameter of the gradient model has been varied by taking $l=2.5 \mathrm{~mm}, l=5.0 \mathrm{~mm}$ and $l=7.5 \mathrm{~mm}$. The results in Figure 12 confirm the analytical solution for the localisation band width (for this problem: $\frac{w}{2}=\pi l$ ). A new stage in the localisation process is entered when in some part of the localisation zone the strength contribution due to local 

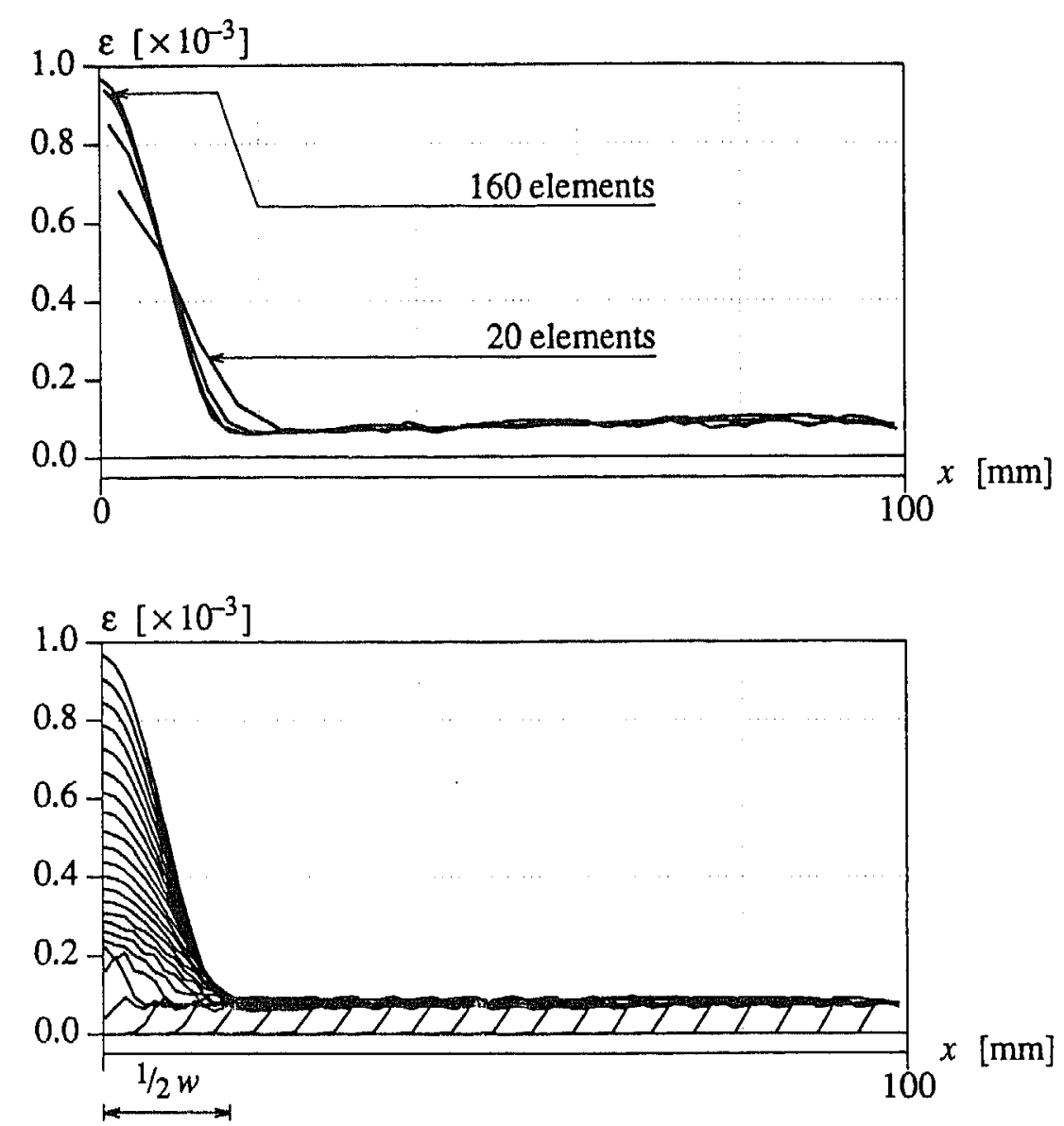

FIGURE 10. Gradient-dependent model with $t_{d}=0$. Top: Strain localisation along the bar at $t=0.2 \cdot 10^{-3} \mathrm{~s}$. Bottom: Development of the localisation band (160 elements).

softening has vanished, so that the load-carrying capacity is only due to gradient effects. The wave length $\lambda$ then starts to increase, the wave speed becomes positive and the localisation zone starts to extend. This phenomenon is plotted in the picture of Figure 13 for an analysis with a slightly different parameter set: $\bar{c}=100000 \mathrm{~N}$ and $h=-4000 \mathrm{~N} / \mathrm{mm}^{2}$.
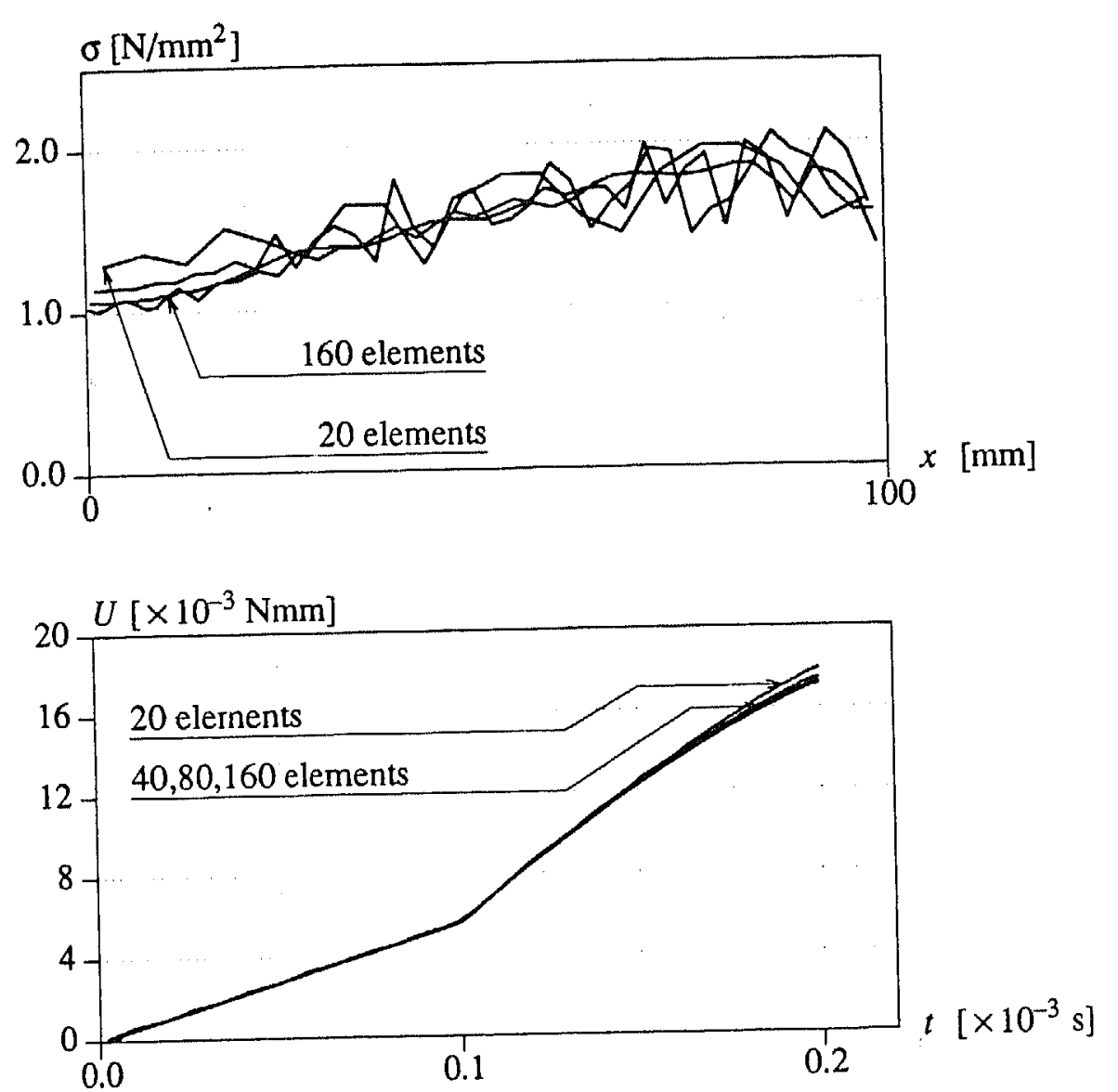

FIGURE 11. Gradient-dependent model with $t_{d}=0$. Top: Stress profiles along the bar at $t=0.2 \cdot 10^{-3} \mathrm{~s}$. Bottom: Energy consumption of the bar.

4 Dispersive Waves in Rate-dependent Softening Plasticity

\subsection{MODEL FORMULATION}

When rate effects are incorporated in the constitutive model, the formulation for the strain-softening function changes into

$$
\sigma=f\left(\varepsilon^{p}, \dot{\varepsilon}^{p}\right)
$$

A simple version, which will be considered here, is a rate-dependent model 


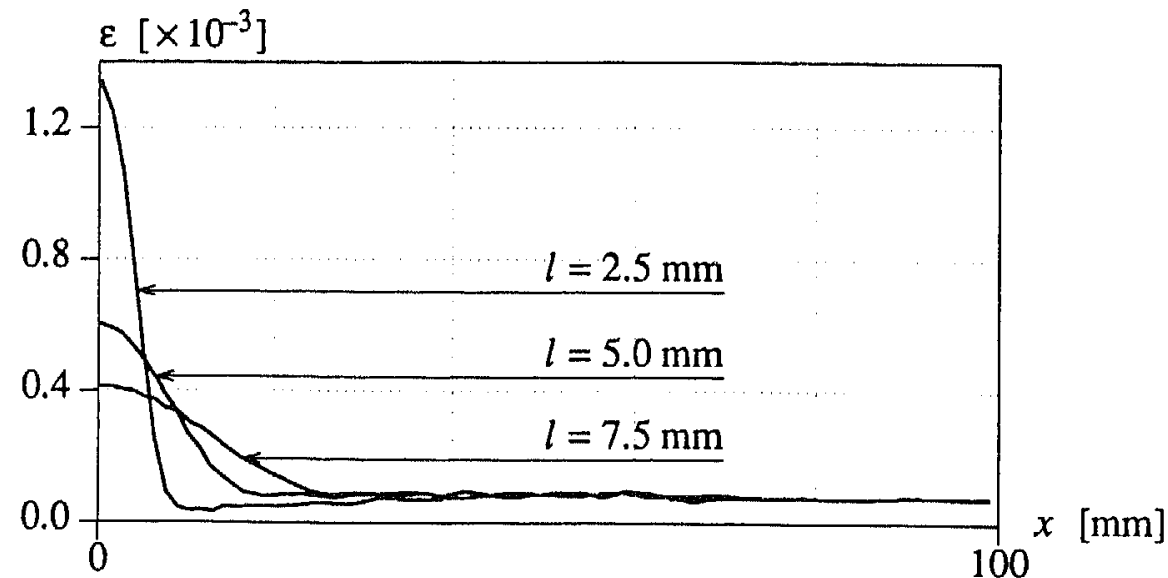

FIGURE 12. Variation of the length scale parameter $\left(160\right.$ elements $\left.-t=0.17 \cdot 10^{-3} \mathrm{~s}\right)$.

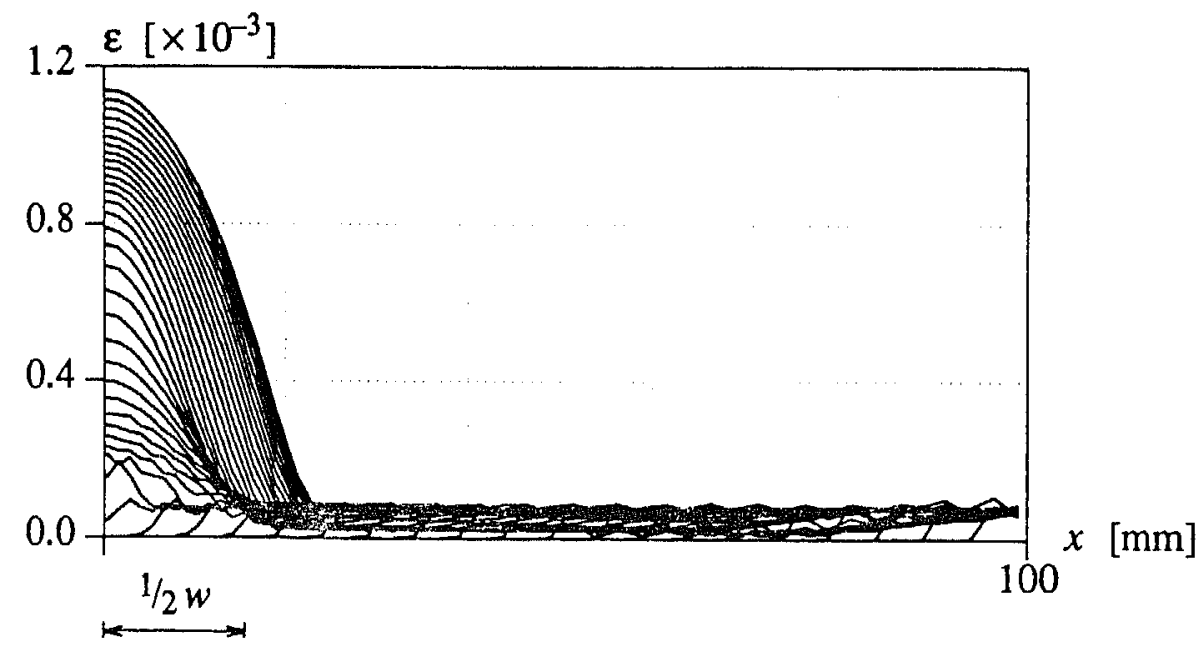

FIGURE 13. Extension of the localisation zone after the termination of softening. that linearly depends on the plastic strain rate. In a rate format we then obtain

$$
\dot{\sigma}=f^{\prime} \dot{\varepsilon}^{p}+m \frac{\partial \dot{\varepsilon}^{p}}{\partial t}
$$

with $m$ is a (constant) rate-sensitivity parameter. When we additionally assume linear softening $\left(f^{\prime}=h=\right.$ constant), we obtain as constitutive relation

$$
\dot{\sigma}=h \dot{\varepsilon}^{p}+m \frac{\partial \dot{\varepsilon}^{p}}{\partial t} .
$$

Substitution of the elastic stress-strain law (4), the decomposition of strain rates (3) and the constitutive equation (55) in the weak form of the equation of motion (1), invoking the divergence theorem and neglecting boundary tractions then gives

$$
\rho \int_{V} \delta v \frac{\partial^{2} v}{\partial t^{2}} \mathrm{~d} V+\int_{V} \delta \dot{\varepsilon} E\left(1-\frac{E}{h+E}\right) \dot{\varepsilon} \mathrm{d} V+\int_{V} \delta \dot{\varepsilon} \frac{m E}{h+E} \frac{\partial \dot{\varepsilon}^{p}}{\partial t} \mathrm{~d} V=0
$$

which is used for the finite element discretisation in section 4.3

\subsection{DISPERSION ANALYSIS}

To investigate wave propagation in a one-dimensional rate-dependent bar we combine the constitutive relation (55) with the kinematic equation (2) the decomposition (3) and the elastic part of the stress-strain relation (4) and differentiate the results with respect to $x$. The result is

$$
\frac{\partial}{\partial x}\left(\frac{m}{E} \frac{\partial \dot{\sigma}}{\partial t}+\frac{E+h}{E} \dot{\sigma}\right)=\frac{\partial^{2}}{\partial x^{2}}\left(h v+m \frac{\partial v}{\partial t}\right)
$$

After combination with the equation of motion (1) we obtain the following third-order differential equation

$$
m\left(\frac{1}{c_{e}^{2}} \frac{\partial^{3} v}{\partial t^{3}}-\frac{\partial^{3} v}{\partial x^{2} \partial t}\right)+\frac{E+h}{c_{e}^{2}} \frac{\partial^{2} v}{\partial t^{2}}-h \frac{\partial^{2} v}{\partial x^{2}}=0
$$

In absence of rate effects $(m \rightarrow 0)$ the classical wave equation $(8)$ is recovered with imaginary characteristics and imaginary wave speeds (compare the 
gradient-dependent bar). We again investigate the type of the wave equation by means of its characteristics. For this purpose we consider the variation of two second-order derivative terms of $v$

$$
\begin{gathered}
d\left(\frac{\partial^{2} v}{\partial t^{2}}\right)=\frac{\partial^{3} v}{\partial t^{3}} \mathrm{~d} t+\frac{\partial^{3} \vartheta}{\partial x \partial t^{2}} \mathrm{~d} x \\
\mathrm{~d}\left(\frac{\partial^{2} v}{\partial x \partial t}\right)=\frac{\partial^{3} v}{\partial x \partial t^{2}} \mathrm{~d} t+\frac{\partial^{3} v}{\partial x^{2} \partial t} \mathrm{~d} x .
\end{gathered}
$$

Combination of eqs. (59), (60) and the wave equation for the rate-dependent bar (eq. 58) yields a system of three third-order differential equations with a characteristic determinant

$$
D=m\left[\left(1 / c_{e}^{2}\right) \mathrm{d} x^{2}-\mathrm{d} t^{2}\right] .
$$

Setting $D=0$ we observe that the characteristics are equal to the elastic bar velocity $\pm c_{e}$ and remain real when strain softening occurs. Accordingly, the wave equation remains hyperbolic and the initial value problem is wellposed.

However, the characteristics are generally not equal to the physical wave speed in the rate-dependent bar. Only when the second-order terms in eq. (58) vanish $(m \rightarrow \infty)$ the wave speed becomes equal to the elastic wave velocity $c_{e}$ as will be proven below by a dispersion analysis. So, the suggestion in literature (Needleman 1988, Loret and Prévost 1990) that in a rate-dependent continuum disturbances due to inelastic effects travel with the elastic wave speed is only correct for the limiting case.

To investigate the dispersive character of wave propagation in the ratedependent, softening continuum a general solution for a single linear harmonic wave with angular frequency $\omega$ and wave number $k$ is assumed to be of a form given by eq. (14). The dispersion relation can be obtained by substitution of (14) into eq. (58). The result is:

$$
\left(\rho m \omega^{3}-m E k^{2} \omega\right) i-\rho(E+h) \omega^{2}+h E k^{2}=0 .
$$

If we consider $\omega$ and $k$ to be real no solution is possible. Eq. (62) can only be satisfied if $k$ is complex, i.e. $k=k_{r}+\alpha i$. This implies that the harmonic wave is attenuated exponentially as it proceeds through the bar. The expression for $v(x, t)$ is now written as

$$
v(x, t)=A e^{-\alpha x} e^{i\left(k_{r} x-\omega t\right)} .
$$

If we equate real and imaginary parts of eq. (62) we obtain

$$
k_{r}^{2}=\frac{\rho \omega^{2}}{2 E}\left(\frac{-\left(m^{2} \omega^{2}+h^{2}+E h\right)+\sqrt{\left.\left(m^{2} \omega^{2}+h^{2}+E h\right)^{2}+(m E \omega)^{2}\right)}}{m^{2} \omega^{2}+h^{2}}\right)
$$

and

$$
\alpha^{2}=\frac{\rho \omega^{2}}{2 E}\left(\frac{+\left(m^{2} \omega^{2}+h^{2}+E h\right)+\sqrt{\left.\left(m^{2} \omega^{2}+h^{2}+E h\right)^{2}+(m E \omega)^{2}\right)}}{m^{2} \omega^{2}+h^{2}}\right)
$$

respectively. In the Figures 14,15 and 16 the results are plotted for the parameter set as listed in Figure 1 and for a value of $m=0.2 \mathrm{Ns} / \mathrm{mm}^{2}$. The dispersion relation $\omega=f\left(k_{r}\right)$ of Figure 14 shows that waves in a rateThe dispersion relation $\omega=f\left(k_{r}\right)$ of Figure 14 shows that is very similar to waves in a linear elastic continuum. If $k_{r}$ approaches zero, i.e. for waves of waves in a linear elastic the slope of the dispersion curve becomes infinite for a which means that the quotient $\omega / k_{r} \rightarrow \infty$ for a static response. As for the gradient-dependent strain-softening bar wave propagation is dispersive since the phase velocity $c_{f}=\omega / k_{r}$ is a function of $\omega$ (Figure 15). For this reason also in the rate-dependent softening continuum the shape of an arbitrary travelling wave can be transformed into a stationary localisation wave.

In Figure 16 the damping coefficient $\alpha$ is plotted as a function of $\omega$. The limit of $\alpha$ with respect to $\omega$ reads

$$
\lim _{\omega \rightarrow \infty} \alpha(\omega)=l^{-1}, \quad \text { with } \quad l=\frac{2 m c_{e}}{E} .
$$

The parameter $l$ sets the internal length scale of this rate-dependent sof tening plasticity model. High frequencies are attenuated exponentially in the space domain to an extent which is determined by the length scale $l$. The implicit presence of an internal length scale is essential for the solution of the mesh-sensitivity problem. 

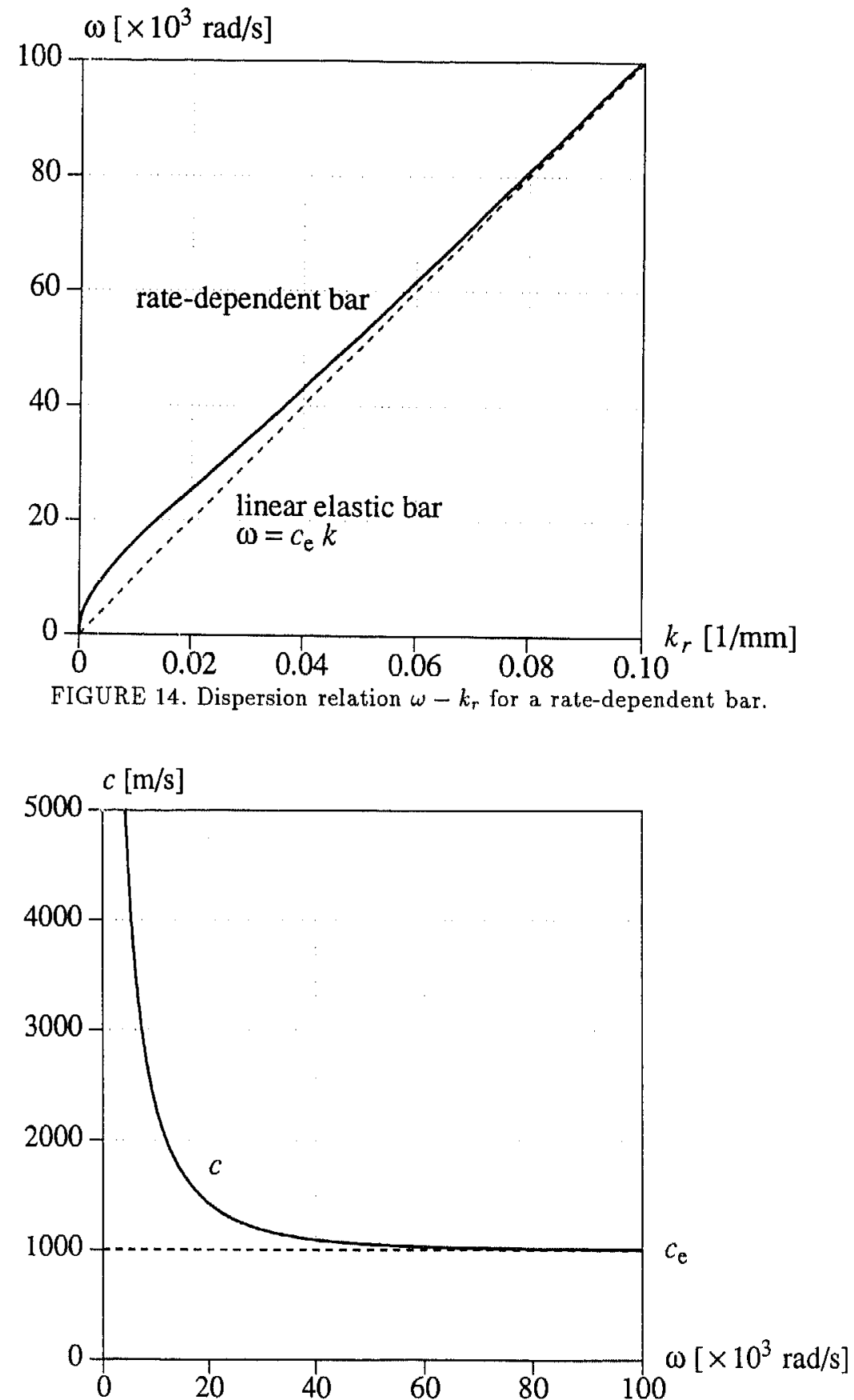

FIGURE 15. Phase velocity as a function of $\omega$ for a rate-dependent bar.
4.3 THE INFLUENCE OF THE DISCRETISATION ON DISPERSIVE WAVES

The dispersion analysis is also carried out for the discretised bar of ratedependent softening material. As shown in section 3.3 the finite element size and the mass distribution can contribute to the dispersive behaviour of waves.

For the rate-dependent model we assume a discretisation of eq. (56) via

$$
\begin{gathered}
v(x)=\mathbf{H} \mathbf{a}, \\
\dot{\varepsilon}(x)=\mathbf{B} \mathbf{a},
\end{gathered}
$$

in which quadratic polynomials have been used for velocity field $v$. Substitution of eqs. (67) and (68) in eq. (56) yields

$$
\int_{V} \rho \mathbf{H}^{T} \mathbf{H} \mathrm{d} V \ddot{\mathbf{a}}+\int_{V} E\left(1-\frac{E}{h+E}\right) \mathbf{B}^{T} \mathbf{B} \mathrm{d} V \mathbf{a}+\int_{V} \frac{m E}{h+E} \frac{\partial \dot{\varepsilon}^{p}}{\partial t} \mathbf{B}^{T} \mathrm{~d} V=0
$$

The matrices in eq. (69) have been determined for a three-noded quadratic bar element of size $d$ with a three-point Gaussian quadrature. We assume the virtual work equation (69) for the centre node $j$ of one element which gives

$$
\frac{M_{d}}{E}+\frac{8}{3 d}\left(1-\frac{E}{h+E}\right)\left(-a_{j-1}+2 a_{j}-a_{j+1}\right)+\frac{0.8607 m}{h+E}\left(\frac{\partial \dot{\varepsilon}_{1}^{p}}{\partial t}-\frac{\partial \dot{\varepsilon}_{3}^{p}}{\partial t}\right)=0
$$

in which $\partial \dot{\varepsilon}_{1}^{p} / \partial t$ and $\partial \dot{\varepsilon}_{3}^{p} / \partial t$ are the quantities in the integration points 1 and 3 , respectively. The parameter $M_{d}$ again represents the mass discretisation in the finite element and for the consistent, the lumped (row-sum technique) and the higher-order mass matrix we obtain

$$
\begin{aligned}
& M_{d}=\frac{\rho d}{15}\left(\frac{\partial^{2} a_{j-1}}{\partial t^{2}}+8 \frac{\partial^{2} a_{j}}{\partial t^{2}}+\frac{\partial^{2} a_{j-1}}{\partial t^{2}}\right) \\
& M_{d}=\frac{2 \rho d}{3} \frac{\partial^{2} a_{j}}{\partial t^{2}}
\end{aligned}
$$




$$
M_{d}=\frac{\rho d}{30}\left(\frac{\partial^{2} a_{j-1}}{\partial t^{2}}+18 \frac{\partial^{2} a_{j}}{\partial t^{2}}+\frac{\partial^{2} a_{j-1}}{\partial t^{2}}\right) .
$$

For the dispersion analysis we assume the exponentially damped harmonic solution according to eq. (63)

$$
\begin{gathered}
a_{j}=A e^{-\alpha x} e^{i\left(k_{r} x-\omega t\right)} \\
\frac{\partial \dot{\varepsilon}_{2}^{p}}{\partial t}=\frac{E\left(k_{r}+\alpha i\right) \omega}{h+E-m i \omega} A e^{-\alpha x} e^{i\left(k_{r} x-\omega \grave{\imath}\right)}
\end{gathered}
$$

and for the neighbouring nodes and integration points we obtain

$$
a_{j-1}(-d / 2)=(\cos k d / 2-i \sin k d / 2) e^{\alpha d / 2} A e^{-\alpha x} e^{i\left(k_{r} x-\omega t\right)}
$$

$$
\begin{aligned}
& \frac{\partial \dot{\varepsilon}_{1}^{p}}{\partial t}(-\sqrt{0.15} d)= \\
& \frac{E\left(k_{r}+\alpha i\right) \omega}{h+E-m i \omega}(\cos \sqrt{0.15} k d-i \sin \sqrt{0.15} k d) e^{\sqrt{0.15} \alpha d} A e^{-\alpha x} e^{i\left(k_{r} x-\omega t\right)}
\end{aligned}
$$

Substitution of the solutions eqs. (74)-(77) in eq. (70) and separation of real and imaginary part yields a system of two equations. A dispersive curve can be derived which exactly doubles back on the continuum curve in Figure 14 and no influence of the finite element size is observed. So, if the dispersion property is determined by a rate-dependent term in the rate boundary value problem there is no influence of the spatial discretisation on the dispersive behaviour of waves.

\subsection{ONE-DIMENSIONAL FINITE ELEMENT ANALYSES}

The strain-softening bar of Figure 1 will now be analysed numerically for the case that rate effects are incorporated. As for the gradient model a slight modification of the parameter set is applied $\left(h=-5000 \mathrm{~N} / \mathrm{mm}^{2}\right)$. This modification does not necessarily increase the brittleness of the material because the viscosity of the material also "carries" a part of the load. The value for the material rate-sensitivity parameter $m=0.2 \mathrm{Ns} / \mathrm{mm}^{2}$, results in a length scale parameter $l=20 \mathrm{~mm}$ (eq.66).
In the first analysis the same loading pulse as in the rate-independent analysis is used $\left(t_{d}=0\right)$. In Figure 17 a comparison between different meshes is made, at a time that the loading pulse has returned at the point of loading. The exponential decrease in strain after reflection that was predicted analytically comes out nicely. The strain pattern of the coarse mesh (10 elements) still deviates somewhat but the finer meshes give identical results and a localisation zone emerges that converges to a finite, constant band width upon mesh refinement. In the bottom part of Figure 17 the development of the total strain along the bar is plotted for time intervals of $1 \cdot 10^{-5} \mathrm{~s}$. The figure shows that the width of the localisation zone remains constant while the loading wave propagates. Mesh independence is not only obtained in the sense that the band width is constant upon mesh refinement but also in the sense that the wave reflection pattern is insensitive with respect to the mesh as can be seen from the stress profiles (Figure 18) of the reflected wave. We observe a partial reflection on the localisation zone, which is constant upon mesh refinement. Finally, the bottom part of Figure 18 shows that the energy consumption remains finite during the loading cycle.

A second analysis has been carried out for a different loading pulse. The loading pulse firstly increases linearly in time before it becomes constant $\left(t_{d}=50 \cdot 10^{-6} \mathrm{~s}\right)$. Again the effect of the inclusion of the length scale can be observed from the strain localisation plots for different meshes and at different times, Figure 19. Note that the strain distribution in the localisation zone has a different shape for this loading case. In the previous analysis a sharp peak in the strain occurs at the left boundary, whereas in this analysis the strain profile is more uniformly distributed and has a lower peak value. This is due to the strain rate profiles in the bar at the moment of plastification. In the previous analysis plastification is initiated in one point at the left boundary from which the exponential decay started. In this analysis the static yield strength is exceeded over a zone with a fixed length $(16.7 \mathrm{~mm})$. At the edge of this zone $(x=16.7 \mathrm{~mm})$ the attenuation of the loading wave starts exponentially and at this point a bending point in the strain profile occurs.

Finally, the width of the localisation band has been analysed. Firstly, the influence of the length scale parameter on the observed localisation width was investigated in an analysis with $t_{d}=0$ by using three different values for $l$, namely 15, 20 and $25 \mathrm{~mm}$. From Figure $20 \mathrm{it}$ appears that the width of the localised zone is a function of the length scale parameter. These results agree with the observation that the localisation zone should vanish when the length scale parameter approaches zero. A comparison of the results shown 


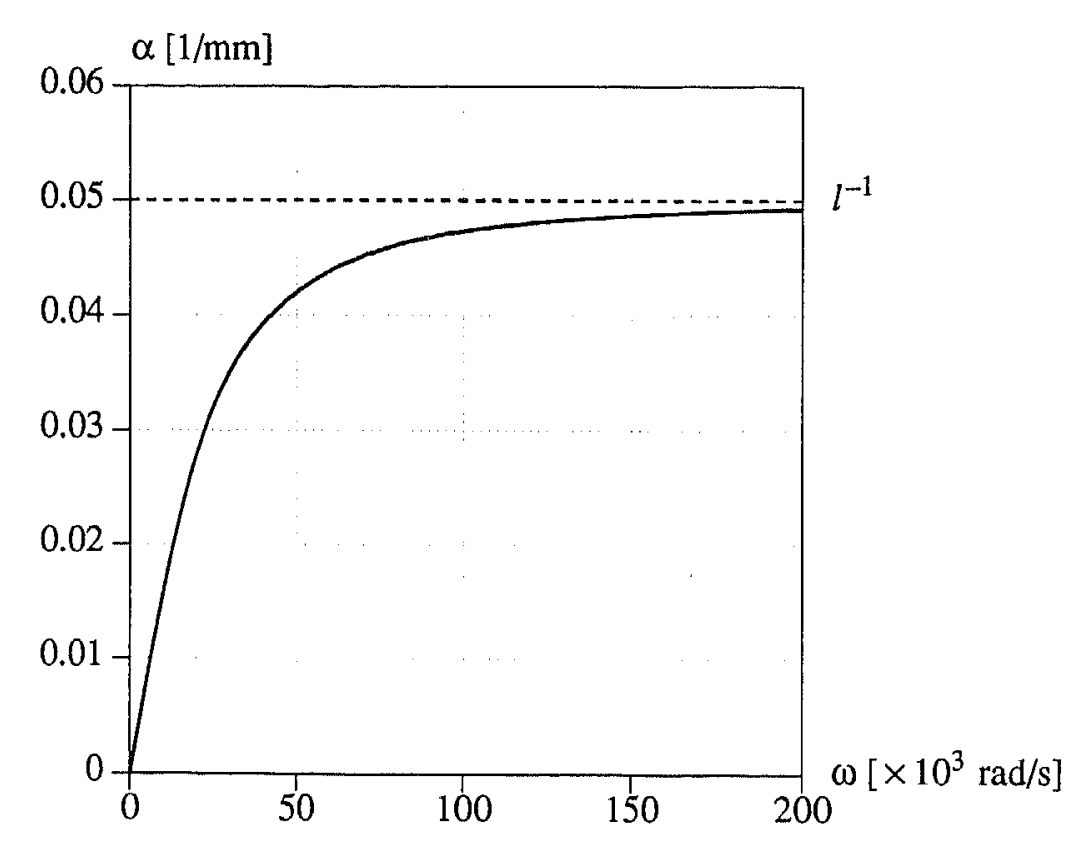

FIGURE 16. Damping coefficient $\alpha$ as a function of $\omega$ for a rate-dependent bar

in the Figures 17 and 19 makes it clear that the shape of the loading wave influences the strain rate distribution in the localisation zone and therefore also the localisation band width. This effect is shown more clearly in the bottom part of Figure 20 where three different values have been considered for the time span $t_{d}$ in which the load is increased from zero to its maximum value.

\section{Concluding Remarks}

In this article the crucial role of dispersion has been emphasised for the formation of stationary localisation waves in dynamically loaded solids. For a non-dispersive continuum localisation necessarily occurs in a set of measure zero, and the direction of the stationary localisation wave in two and threedimensional numerical simulations is then fully determined by the lay-out of the grid lines (Sluys 1992).

For two enhanced continuum models, namely a model enriched with higherorder strain gradients and a linear rate-dependent model, the dispersion properties have been investigated for one-dimensional wave propagation. This has been done for the continuum model and for the discretised model.
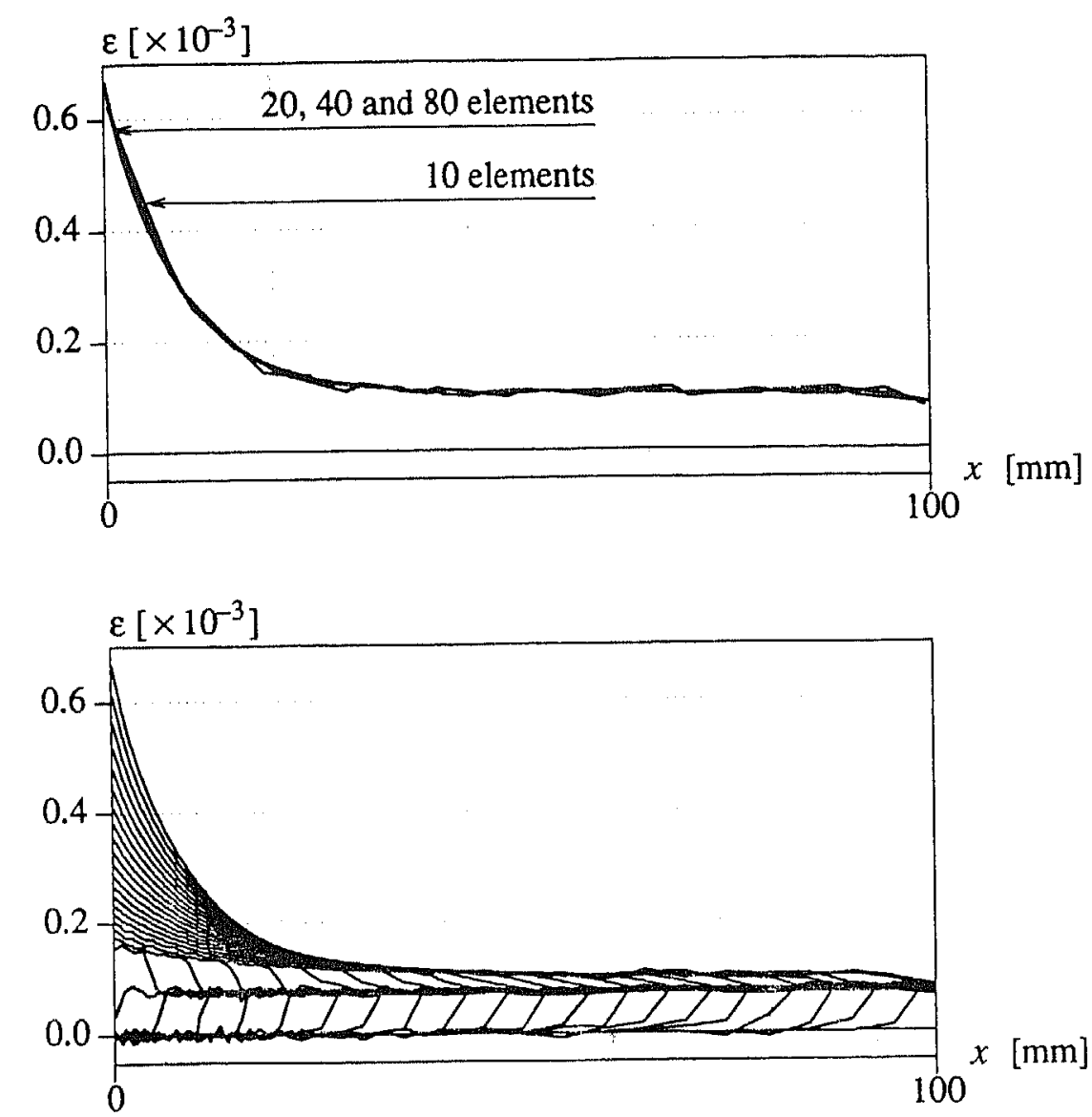

FIGURE 17. Rate-dependent model with $t_{d}=0$. Top: Strain localisation along the bar at $t=0.2 \cdot 10^{-3} \mathrm{~s}$. Bottom: Development of the localisation band (80 elements).

The analytical findings perfectly match the numerical experiments.

\section{Acknowledgements}

Financial support of the Royal Netherlands Academy of Arts and Sciences to the first author is gratefully acknowledged. 
$\sigma\left[\mathrm{N} / \mathrm{mm}^{2}\right]$
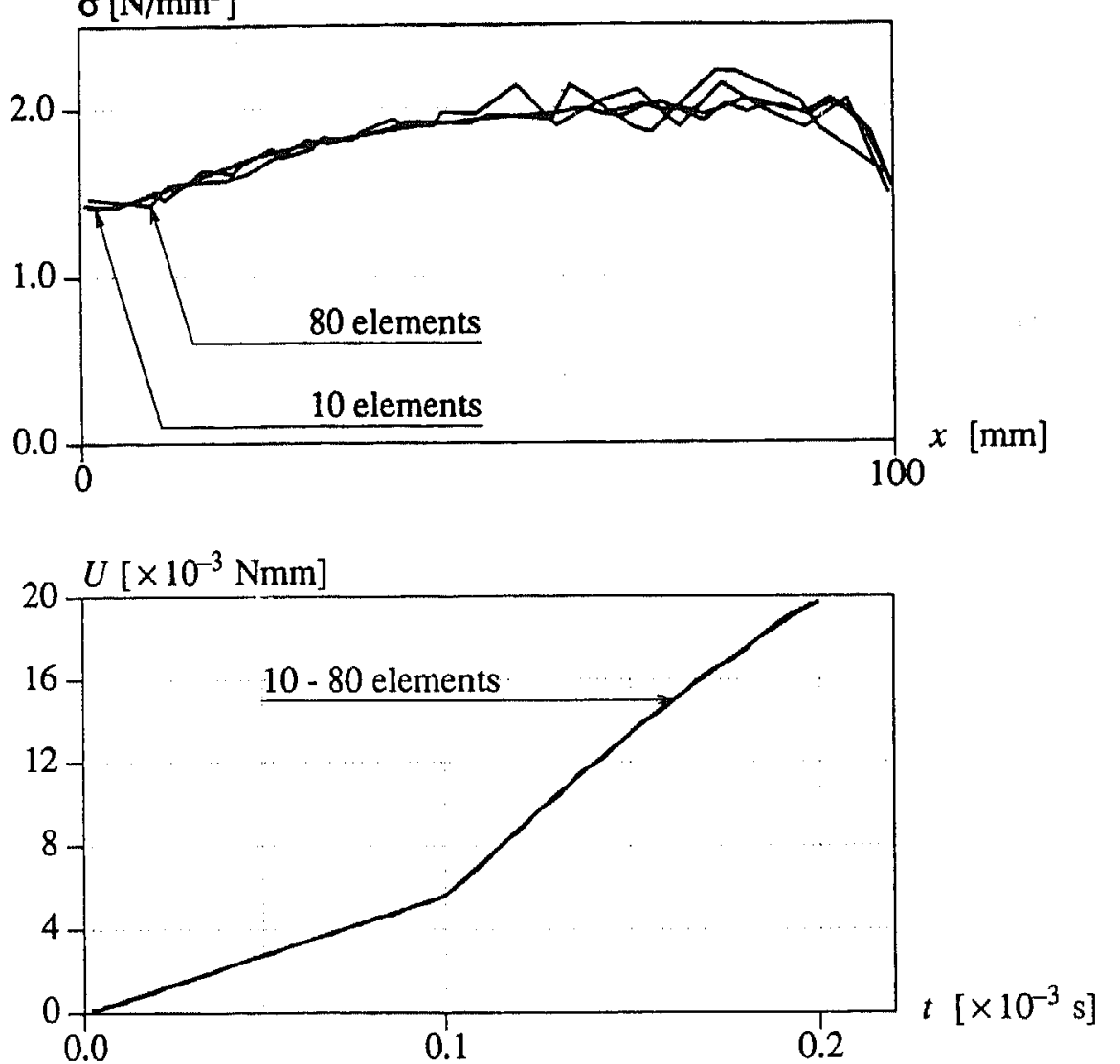

FIGURE 18. Rate-dependent model with $t_{d}=0$. Top: Stress profiles along the bar at $t=0.2 \cdot 10^{-3} \mathrm{~s}$. Bottom: Energy consumption of the bar.

\section{References}

Aifantis, E.C.: On the micro-structural original of certain inelastic models, J. Eng. Mater. Technol., 106, 326-334 (1984).

Bažant, Z.P. and Belytschko, T.: Wave propagation in a strain-softening bar - exact solution, ASCE J. Engng. Mech., 111, 381-389 (1985).

Benallal, A., Billardon, R. and Geymonat, G.: Localization phenomena at the boundaries and interfaces of solids, in Proc. Third Int. Conf. Constitutive Laws for Engineering Materials: Theory and Applications, (Ed. Desai, C.S.), pp. 387-390, Tucson, Arizona (1991).
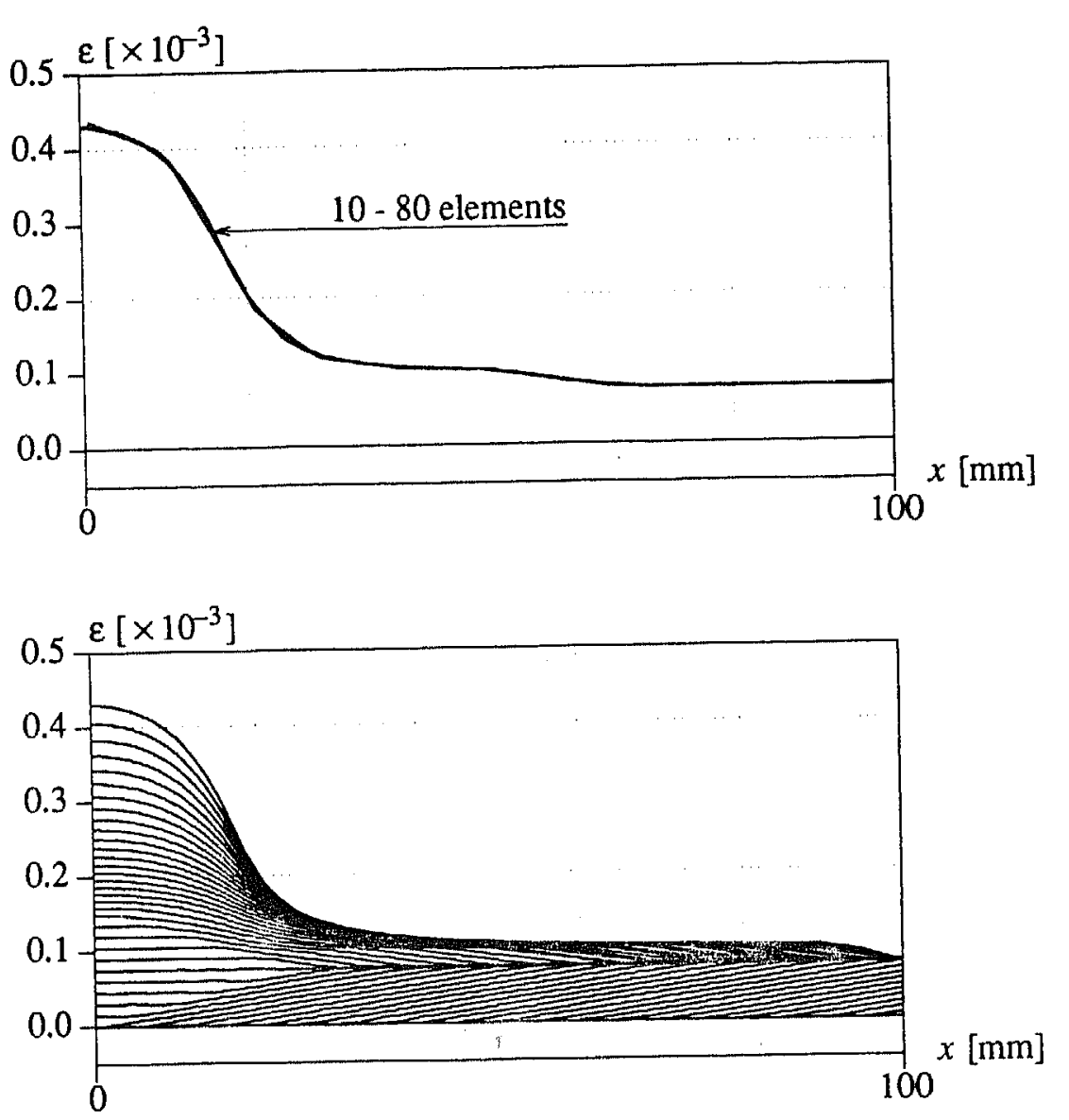

FIGURE 19. Rate-dependent model with $t_{d}=50 \cdot 10^{-6} \mathrm{~s}$. Top: Strain localisation along the bar at $t=0.25 \cdot 10^{-3} \mathrm{~s}$. Bottom: Development of the localisation band ( 80 elements)

Borst, R. de: Simulation of strain localisation: A reappraisal of the Cosserat continuum, Eng. Comput., 8, 317-332 (1991a).

Borst, R. de: Numerical modelling of bifurcation and localisation in cohesivefrictional materials, PAGEOPH, 136, 367-390 (1991b).

Borst, R. de and Sluys, L.J.: Localisation in a Cosserat continuum under static and dynamic loading conditions, Comp. Meth. Appl. Mech. Eng., 90, 805-827 (1991)

Borst, R. de: A generalisation for $J_{2}$-flow theory for polar continua, Comp. Meth. Appl. Mech. Eng., (1992) to appear.

Borst, R. de and Mühlhaus, H.-B.: Gradient-dependent plasticity: Formula- 


\section{$\varepsilon\left[\times 10^{-3}\right]$}
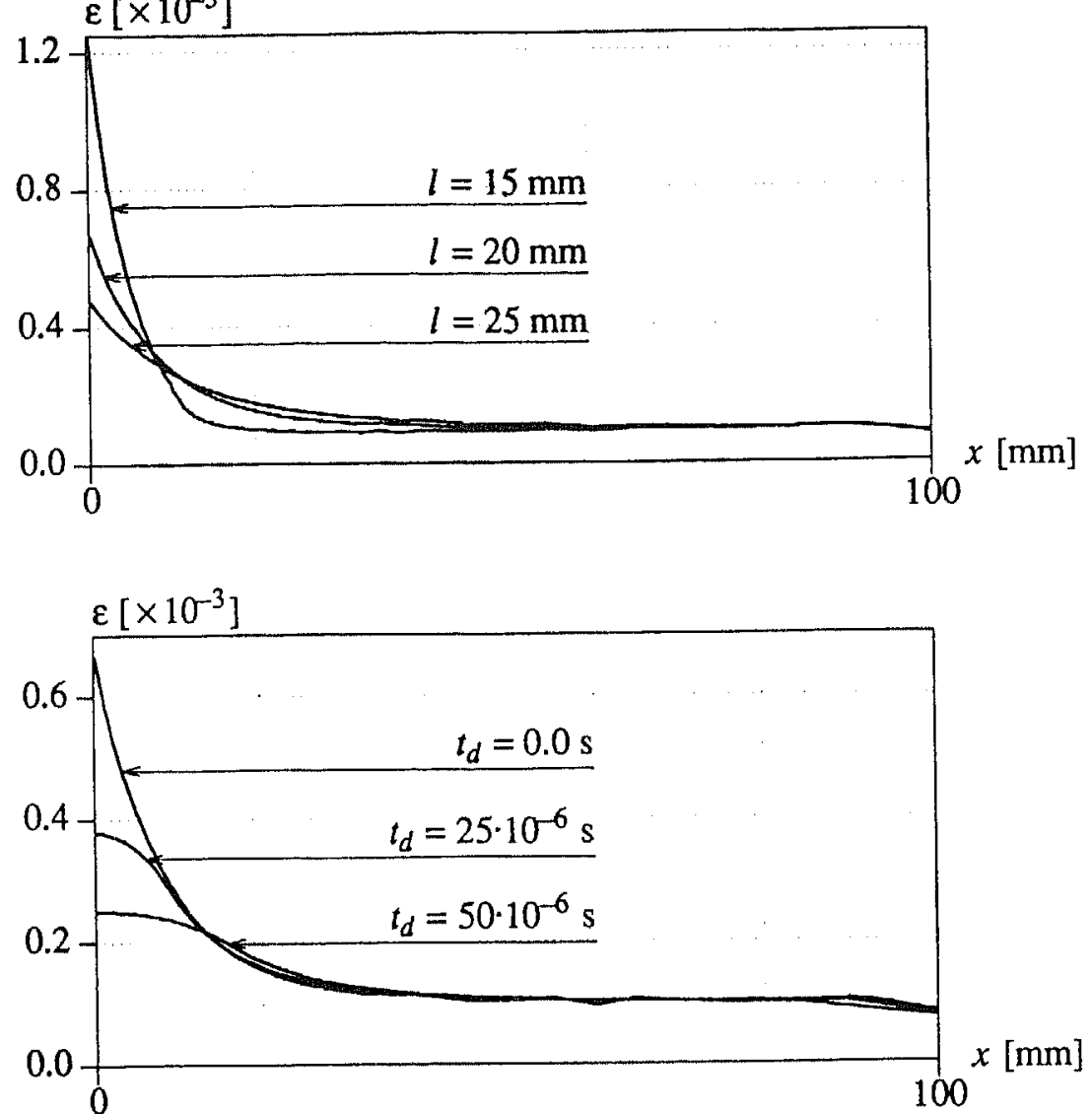

FIGURE 20. Rate-dependent model. Top: Variation of the length scale parameter $\left(t=0.2 \cdot 10^{-3} \mathrm{~s}\right.$.). Bottom: Variation of the loading rate $\left(t=0.2 \cdot 10^{-3} \mathrm{~s}\right.$. $)$.

tion and algorithmic aspects, Int. J. Num. Meth. Eng., 35, 521-539 (1992). Coleman, B.D. and Hodgdon, M.L.: On shear bands in ductile materials, Arch. Ration. Mech. Anal., 90, 219-247 (1985)

Eringen, A.C.: Linear theory of nonlocal elasticity and dispersion of plane waves, Int. J. Engng. Sci., 10, 425-435 (1972).

Eringen, A.C.: Nonlocal elasticity and waves, in Continuum Mechanics Aspects of Geodynamics and Rock Fracture Mechanics (Ed. Thoft-Christensen), pp. 81-105, D. Reidel Publishing Company, Dordrecht (1974).

Huerta, A. and Pijaudier-Cabot, G.: Discretisation influence on the regularization by two localization limiters, in Numerical Methods in Engineering
'92 (Ed. Hirsch,Ch., Zienkiewicz,O.C. and Oñate,E.), 65-72, Elsevier, Amsterdam and London (1992).

Hughes, T.J.R.: The Finite Element Method. Linear Static and Dynamic Finite Element Analysis.Prentice-Hall, New Jersey (1987).

Lasry, D. and Belytschko, T.: Localization limiters in transient problems, Int. J. Solids Structures, 24, 581-597 (1988).

Loret, B. and Prévost, J.H.: Dynamic strain localization in elasto-(visco-)plastic solids, Part 1. Comp. Meth. Appl. Mech. Engng., 83, 247-273 (1990).

Mier, J.G.M. van: Strain softening of concrete under multiaxial loading conditions, Dissertation, Eindhoven University of Technology (1992).

Mühlhaus, H.-B.: Application of Cosserat theory in numerical solutions of limit load problems, Ingenieur-Archiv, 59, 124-137 (1989).

Mühlhaus, H.-B., Borst, R. de and Aifantis, E.C.: Constitutive models and numerical analyses for inelastic materials with microstructure, in Computer Methods and Advances in Geomechanics, (Eds. Beer, G., Booker, J.R. and Carter, J.P.), pp. 377-386, Balkema, Rotterdam and Boston (1991).

Mühlhaus, H.-B. and Aifantis, E.C.: A variational principle for gradient plasticity, Int. J. Solids Structures, 19, 845-858 (1991).

Needleman, A.: Material rate dependence and mesh sensitivity in localization problems, Comp. Meth. Appl. Mech. Eng., 67, 69-86 (1988).

Pijaudier-Cabot, G. and Bažant, Z.P.: Nonlocal darnage theory, ASCE J. Engng. Mech., 113, 1512-1533 (1987).

Read, H. and Hegemier, G.: Strain softening of rock, soil and concrete - A review article, Mech. Mater. 3, 271-294 (1984).

Schreyer, H.L. and Chen, Z.: One-dimensional softening with localization, J. Appl. Mech. 53, 791-979 (1986).

Sluys, L.J.: Wave propagation, localisation and dispersion in softening solids, Dissertation, Delft University of Technology (1992).

Sluys, L.J. and Borst, R. de: Wave propagation and localisation in a ratedependent cracked medium: Model formulation and one-dimensional examples, Int. J. Solid Structures, 29,2945-2958 (1992).

Whitham,G.B.: Linear and nonlinear Waves. Wiley, London and New York (1974). 